\title{
On Correspondences between Once Punctured Tori and Closed Tori: Fricke Groups and Real Lattices
}

\author{
Ryuji ABE
}

Keio University

\section{Introduction.}

We consider the Teichmüller space of the closed torus and the Teichmüller space of the once punctured torus. It is well-known that the former can be identified with the upper halfplane and that several coordinate systems can be introduced to the latter. This is the first part of a series of papers in which we investigate explicit relations between these two Teichmüller spaces. In this paper based on a correspondence of subsets of these spaces we will give an explicit construction of a holomorphic mapping between a once punctured torus and a closed torus.

We use throughout the convention that an element $A$ in $\operatorname{PSL}(2, \mathbf{R})$ represents the Möbius transformation induced by $A$, i.e.,

$$
\text { if } A=\left(\begin{array}{ll}
a & b \\
c & d
\end{array}\right) \in \operatorname{PSL}(2, \mathbf{R}) \text { then } A(z)=\frac{a z+b}{c z+d} .
$$

We consider a Fuchsian group $G$ consisting of Möbius transformations of $\operatorname{PSL}(2, \mathbf{R})$ and having the following properties: (i) $G$ is discontinuous in the upper half-plane $\mathbf{H}$, (ii) every real number is a limit point for $G$, (iii) $G$ is finitely generated.

Definition 1.1. A Fuchsian group $\Gamma=\langle A, B\rangle$ for $A, B \in \operatorname{PSL}(2, \mathbf{R})$ is called a Fricke group if $A, B$ are hyperbolic and $\operatorname{tr}\left[B^{-1}, A^{-1}\right]=-2$.

In the definition above $\Gamma=\langle A, B\rangle$ is the free group generated by $A, B$ and tr denotes the trace of a matrix. We consider a once punctured torus which is uniformized by a Fricke group $\Gamma$ and take a normalized form for the presentation of $\Gamma$ (see §5). By using the quantities $X=\operatorname{tr} A, Y=\operatorname{tr} B$ and $Z=\operatorname{tr} A B$, the above description of the Fricke group is characterized by $X^{2}+Y^{2}+Z^{2}=X Y Z$ and $X, Y, Z>2$. Moreover, we obtain the following theorem (see [W]).

THEOREM 1.1 (Fricke [F], Keen [K]). The Teichmüller space $\mathcal{T}_{1,1}$ of the once punctured torus is the sublocus of $X^{2}+Y^{2}+Z^{2}=X Y Z$ with $X, Y, Z>2$. 
In this paper we denote a point in the Teichmüller space $\mathcal{T}_{1,1}$ of the once punctured torus by a triplet $(X, Y, Z)$. We call this triplet the $(X, Y, Z)$ coordinates.

We describe a closed torus by $R_{\tau}=\mathbf{C} / \Gamma_{\tau}, \Gamma_{\tau}=\{m+n \tau \mid m, n \in \mathbf{Z}\}$, then the Teichmüller space $\mathcal{T}_{1,0}$ of the closed torus is the upper half-plane $\mathbf{H}$, i.e., a point in the Teichmüller space $\mathcal{T}_{1,0}$ of the closed torus is denoted by $\tau \in \mathbf{H}$ (See, for example, [IT]). We call $\tau$ representing a closed torus the $\tau$ coordinates.

It is well-known that theoretically we can identify Teichmüller spaces $\mathcal{T}_{1,0}$ and $\mathcal{T}_{1,1}$. For example in [W] the existence of a map from the $(X, Y, Z)$ coordinates to the $\tau$ coordinates is described. But it does not give explicitly a holomorphic mapping between a once punctured torus determined by $(X, Y, Z)$ and a closed torus determined by $\tau$. Our problem is to construct such a holomorphic mapping.

B. Maskit gave in [Mas1] and [Mas2] the existence of a conformal embedding from a once punctured torus to a closed torus and correspondences among good subsets of $\mathcal{T}_{1,0}$ and $\mathcal{T}_{1,1}$ by using another parametrization of Fuchsian groups. Now we state these correspondences in our context. In order to introduce good subsets of $\mathcal{T}_{1,0}$ we consider the action of the Teichmüller modular group. It is well-known that the Teichmüller modular group for $\mathcal{T}_{1,0}$ is $\operatorname{SL}(2, \mathbf{Z})$ and a fundamental domain for the action of $\operatorname{PSL}(2, \mathbf{Z})$ on $\mathcal{T}_{1,0}$ can be represented by $F=\{\tau \in \mathbf{H}|| \tau \mid \geq 1$ and $|\operatorname{Re}(\tau)| \leq 1 / 2\}$. Moreover, we introduce the following subsets of $F: L_{1}=\{\tau \in \mathbf{H}|| \tau \mid \geq 1$ and $\operatorname{Re}(\tau)=0\}, L_{2}=\{\tau \in \mathbf{H}|| \tau \mid=1$ and $-1 / 2 \leq \operatorname{Re}(\tau) \leq 0\}$, $L_{3}=\{\tau \in \mathbf{H}|| \tau \mid \geq 1$ and $\operatorname{Re}(\tau)=-1 / 2\}$. These sets are characterized by the fact that in $F, \tau \in L_{1} \cup L_{2} \cup L_{3}$ if and only if for some $\mu \in \mathbf{C}$ the lattice $\mu \Gamma_{\tau}$ is a real lattice, that is, $\overline{\mu \Gamma_{\tau}}=\left\{\overline{\mu \gamma} \mid \mu \gamma \in \mu \Gamma_{\tau}\right\}=\mu \Gamma_{\tau}$ (See $\left.\$ 2\right)$.

It is also well-known that the Teichmüller modular group $\mathcal{M}$ for $\mathcal{T}_{1,1}$ is isomorphic to $\operatorname{SL}(2, \mathbf{Z})$. Let $\mathcal{P} \mathcal{M}$ be the quotient group of $\mathcal{M}$ by the kernel of the action of $\mathcal{M}$ on $\mathcal{T}_{1,1}$. Then a fundamental domain for the action of $\mathcal{P} \mathcal{M}$ on $\mathcal{T}_{1,1}$ is given by $M=\{(X, Y, Z) \in$ $\left.\mathcal{T}_{1,1} \mid 2<X \leq Y \leq Z \leq X Y / 2\right\}$ (See $\$ 3$ ). The correspondences of subsets can be described as follows:

THEOREM 1.2. We define three subsets of $M: M_{1}=\{(X, Y, Z) \in M \mid Z=X Y / 2\}$, $M_{2}=\{(X, Y, Z) \in M \mid X=Y\}, M_{3}=\{(X, Y, Z) \in M \mid Y=Z\}$. Then there exist the correspondences of the sets: $L_{1} \Leftrightarrow M_{1}, L_{2} \Leftrightarrow M_{2}, L_{3} \Leftrightarrow M_{3}$.

COROLLARY 1.1. The point $i$ corresponds to $(2 \sqrt{2}, 2 \sqrt{2}, 4)$ and the point $\rho=e^{2 \pi i / 3}$ corresponds to $(3,3,3)$.

Based on this correspondence we will give in this paper a construction of a holomorphic mapping between elements of $L_{1}$ and $M_{1}$. Holomorphic mappings between elements of $L_{k}$ and $M_{k}, k=2,3$ will be constructed in [Ab1]. The reasons are as follows. On the one hand an element in $L_{1}$ determines a rectangular lattice and we can take a hexagonal fundamental domain identified with an element in $M_{1}$ (see \$5). Both the rectangular lattice and the hexagonal fundamental domain are simple and easy to investigate. On the other hand an element in $L_{k}$ determines a rhombic lattice and a fundamental domain identified with an element in $\boldsymbol{M}_{k}$ is an octagon. Then we need to investigate the octagonal fundamental domain in detail and to 
use another technique in order to construct our aimed holomorphic mappings. In consequence we obtain two different relations giving holomorphic mappings between elements of $L_{1}$ and $M_{1}, L_{k}$ and $M_{k}$, respectively.

$\mathrm{H}$. Cohn introduced in [C1] a relation giving a holomorphic mapping from the once punctured torus $(3,3,3)$ to the closed torus $\rho$. A basic idea for finding this relation is an abelianization of the Fricke group. We introduce notations in order to recall the relation and give another example. Let $z$ represent a point in the upper half-plane $\mathbf{H}$ and $u$ a point in the complex plane $\mathbf{C}$. We call $\mathbf{H}$ the $z$-plane and $\mathbf{C}$ the $u$-plane. Then a once punctured torus $(X, Y, Z)$ and a closed torus $\tau$ can be identified with fundamental domains in the $z$-plane and the $u$-plane, respectively. A holomorphic mapping from $(3,3,3)$ to $\rho$ is given by

$$
1-J(z)=\wp^{\prime}(u)^{2}=4 \wp(u)^{3}+1,
$$

and a holomorphic mapping from $(2 \sqrt{2}, 2 \sqrt{2}, 4)$ to $i$ is given by

$$
J_{i}(z)=\wp(u)^{2} \text { and } \wp^{\prime}(u)^{2}=4 \wp(u)^{3}-4 \wp(u),
$$

where $\wp(u)$ are the Weierstrass $\wp$-functions defined by the above equations, $J(z)$ is the modular function and $J_{i}(z)$ is a function having similar properties to $J(z)$ (see Proposition 4.1). We will show in $\S 4$ why these relations (1.1) and (1.2) give holomorphic mappings between once punctured tori and closed tori.

Our main result in this paper is described as follows:

THEOREM 1.3. For any $(X, Y, Z) \in M_{1}$ there uniquely exists an element $\tau \in L_{1}$ satisfying the following conditions: if $\tau \in L_{1}$ then $p(x)=4 x^{3}-g_{2}(\tau) x-g_{3}(\tau)$ has three distinct real roots and a holomorphic mapping between $(X, Y, Z)$ and $\tau$ is given by the relation

$$
\wp(u)=\left(x_{2}-x_{1}\right) J_{(X, Y, Z)}(z)+x_{2},
$$

where $x_{1}<x_{2}<x_{3}$ are the three real roots of $p(x), \wp(u)$ is the Weierstrass $\wp$-function defined by $\wp^{\prime}(u)^{2}=4 \wp(u)^{3}-g_{2}(\tau) \wp(u)-g_{3}(\tau)$ and $J_{(X, Y, Z)}(z)$ is a function having similar properties to the modular function $J(z)$.

In $\S 5$ we will give the precise definition of $J_{(X, Y, Z)}(z)$ (Proposition 5.1) and a proof of this theorem.

The holomorphic mapping from $(3,3,3)$ to $\rho$ was used in [C1] in order to investigate Markoff's minimal form which is closely related to geodesics on a once punctured torus. In [C2] it is also suggested that the relations (1.1) and (1.2) can be applied to some kind of word problem considered in associated Fricke groups. Therefore there is a possibility of applying our results to these problems. In particular it is interesting to use the relation (1.3) for coding of geodesics on once punctured tori, because the space $\mathbf{C}$ is much easier to see than the space $\mathbf{H}$. Moreover, the investigation of explicit relations between Teichmüller spaces of once punctured and closed tori is important from the point of view of rigidity problem by Ratner [R], Margulis [Mar], Zimmer [Z], etc., because a Fricke group is a typical example of a discrete subgroup of $\operatorname{SL}(2, \mathbf{R})$ whose $\mathbf{R}$-rank is equal to 1 and a once punctured torus is the simplest surface where geodesic flow and horocycle flow are defined. We can consider 


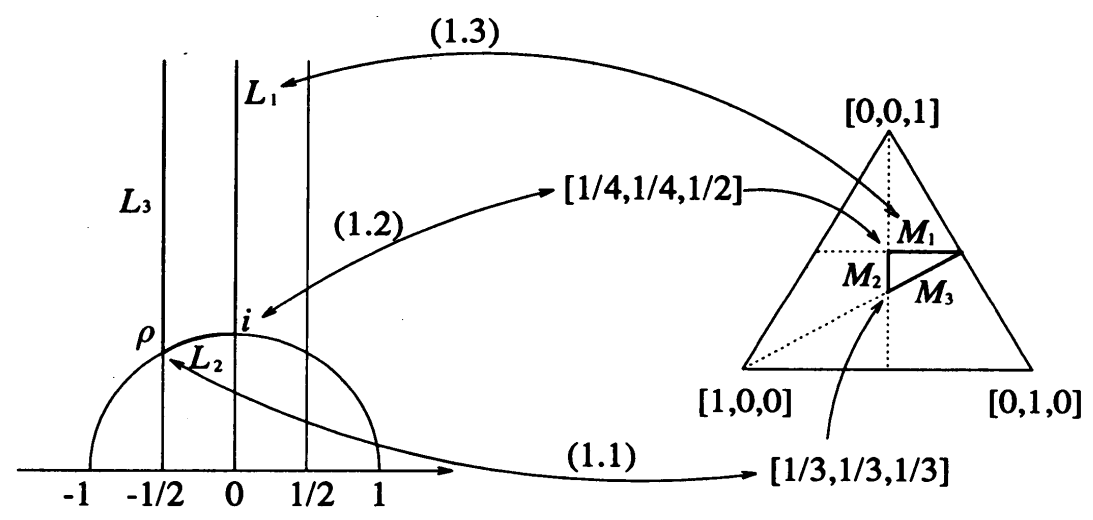

The coordinate system $[a, b, c]$ is defined in $\S 3$. The points $(3,3,3)$ and $(2 \sqrt{2}, 2 \sqrt{2}, 4)$ are identified with $[1 / 3,1 / 3,1 / 3]$ and $[1 / 4,1 / 4,1 / 2]$, respectively.

FIGURE 1.1

the following problems: How can we determine the images of geodesic and horocycle flows under a holomorphic mapping from a once punctured torus to a closed torus? Is the rigidity of horocycle flows preserved under this mapping or not? These are the questions which make it meaningful to construct explicit holomorphic mappings between once punctured and closed tori.

Finally, we give an important remark. Let $\Gamma=\langle A, B\rangle$ be a Fricke group. A construction of a holomorphic mapping from a once punctured torus uniformized by $\Gamma$ to an element in $\mathcal{T}_{1,0}$ is closely related to a construction of a cusp form of weight 1 for $\Gamma$. The existence of such a cusp form is guaranteed; however, we cannot use standard ways of constructing cusp forms, since, for example, a Poincaré series whose denominator is of order 2 does not converge. For this reason we generalized the relation (1.1) and obtained Theorem 1.3. As we mentioned above, the relations (1.1), (1.2) and (1.3) give cusp forms of weight 1 for the Fricke groups corresponding to $(3,3,3),(2 \sqrt{2}, 2 \sqrt{2}, 4)$ and $(X, Y, Z) \in M_{1}$, respectively. Moreover, we can obtain explicit representations of these cusp forms. For more detailed discussion on this point we refer the reader to [Ab2].

ACKNOWLEDGMENTS. I would like to thank P. Arnoux for useful conversations and Y. Ito, Y. Maeda and H. Nakada for various assistance rendered during the course of preparation of this article. The author is grateful to $T$. Schmidt for obtaining information on the existence of the work [S] and to the referee for the existence of the works of Maskit [Mas1] and [Mas2]. I am deeply indebted to the Institut de Mathematiques de Luminy (Marseille) for their hospitality.

\section{Preliminaries.}

In this section we recall fundamental facts concerning Weierstrass $\wp$-function and the modular function. For detailed arguments and proofs we refer the reader to Chapters 3 and 6 
of [JS]. These facts will be used in $\S 4$ and $\S 5$. For our construction of a holomorphic mapping between a closed torus and a once punctured torus, Weierstrass $\wp$-function plays an important role, because this function is defined by using the lattice which determines a closed torus.

For $\omega_{1}, \omega_{2} \in \mathbf{C}$, we define a lattice $\Omega$ by

$$
\Omega=\Omega\left(\omega_{1}, \omega_{2}\right)=\left\{n_{1} \omega_{1}+n_{2} \omega_{2} \mid n_{1}, n_{2} \in \mathbf{Z}\right\},
$$

and we call $\left\{\omega_{1}, \omega_{2}\right\}$ a basis for $\Omega$ and a point in $\Omega$ a lattice point in $\mathbf{C}$.

DEFINITION 2.1. We call the function defined by the following series Weierstrass $\wp-$ function

$$
\wp(u)=\frac{1}{u^{2}}+\sum_{\omega \in \Omega, \omega \neq 0}\left(\frac{1}{(u-\omega)^{2}}-\frac{1}{\omega^{2}}\right) \text { for all } u \in \mathbf{C} .
$$

From the definition, it follows immediately that $\wp(u)$ depends on the lattice $\Omega$ and is an even function which is analytic on $\mathbf{C} \backslash \Omega$ and has a pole of order 2 at each lattice point. Note that $\wp(u)$ is doubly periodic with respect to the lattice $\Omega$. We define

$$
g_{2}=g_{2}(\Omega)=60 \sum_{\omega \in \Omega, \omega \neq 0} \frac{1}{\omega^{4}} \text { and } g_{3}=g_{3}(\Omega)=140 \sum_{\omega \in \Omega, \omega \neq 0} \frac{1}{\omega^{6}} .
$$

If $\Omega=\Omega(1, \tau)$ they can be written in the following forms:

$$
g_{2}=g_{2}(\tau)=60 \sum_{(m, n) \neq(0,0)} \frac{1}{(m+n \tau)^{4}} \quad \text { and } \quad g_{3}=g_{3}(\tau)=140 \sum_{(m, n) \neq(0,0)} \frac{1}{(m+n \tau)^{6}} .
$$

We can characterize values of $g_{2}(\tau)$ and $g_{3}(\tau)$ when $\tau$ satisfies certain conditions.

PRoposition 2.1. (i) If $\operatorname{Re}(\tau)=t / 2$ for $t \in \mathbf{Z}$, then $g_{2}(\tau)$ and $g_{3}(\tau)$ are real.

(ii) $g_{2}(\rho)=g_{3}(i)=0$ and $g_{2}(i), g_{3}(\rho)$ are real and positive, where $\rho=e^{2 \pi i / 3}$.

In this paper the notation $\rho$ always denotes the complex number $e^{2 \pi i / 3}$. Moreover, we can give characterizations of the function $\wp(u)$ and the lattice $\Omega$ when $g_{2}(\Omega)$ and $g_{3}(\Omega)$ are real. We represent by $\bar{z}$ the conjugate of $z \in \mathbf{C}$.

Proposition 2.2. The following conditions are equivalent:

(i) $g_{2}(\Omega), g_{3}(\Omega) \in \mathbf{R}$.

(ii) $\wp$ defined by using the lattice $\Omega$ satisfies $\wp(\bar{u})=\overline{\wp(u)}$ for all $u \in \mathbf{C}$.

(iii) $\Omega$ is a real lattice, i.e., $\bar{\Omega}=\{\bar{\omega} \mid \omega \in \Omega\}=\Omega$.

In order to characterize real lattices we introduce the following sets:

$$
\begin{aligned}
& L_{1}=\{\tau \in \mathbf{H}|| \tau \mid \geq 1 \text { and } \operatorname{Re}(\tau)=0\}, \\
& L_{2}=\left\{\tau \in \mathbf{H}|| \tau \mid=1 \text { and }-\frac{1}{2} \leq \operatorname{Re}(\tau) \leq 0\right\}, \\
& L_{3}=\left\{\tau \in \mathbf{H}|| \tau \mid \geq 1 \text { and } \operatorname{Re}(\tau)=-\frac{1}{2}\right\} .
\end{aligned}
$$

Let $\tau$ be an element in $L_{1} \cup L_{3}$. If we take $\mu>0$ or $\mu=r i$ with $r>0$, then $\Omega=\Omega(\mu, \mu \tau)$ is a real lattice. Let $\tau=e^{i \theta}$ with $\pi / 2 \leq \theta \leq 2 \pi / 3$ be an element in $L_{2}$. Then $\Omega=\Omega(\mu, \mu \tau)$ 
is a real lattice, where $\mu=r e^{i(\pi-\theta) / 2}$ or $\mu=r e^{-\theta i / 2}$ with $r>0$. Conversely, any real lattice $\Omega$ can be represented as above, because real lattices are classified into two cases: one is a rectangular case, i.e., $\tau \in L_{1}$ and the other is a rhombic case, i.e., $\tau \in L_{2} \cup L_{3}$ (See Fig. 2.1).

DEFINITION 2.2. The modular function $J(\tau)$ is defined by

$$
J(\tau)=\frac{g_{2}(\tau)^{3}}{g_{2}(\tau)^{3}-27 g_{3}(\tau)^{2}} \quad \text { for all } \tau \in \mathbf{H} .
$$

The properties of the modular function which we will use are the following:

PROPOSITION 2.3. (i) $J$ is invariant under the action of the modular group PSL(2,Z), i.e., $J(T(\tau))=J(\tau)$ for all $\tau \in \mathbf{H}$ and $T \in \operatorname{PSL}(2, \mathbf{Z})$, where $T(\tau)$ is a Möbius transformation.

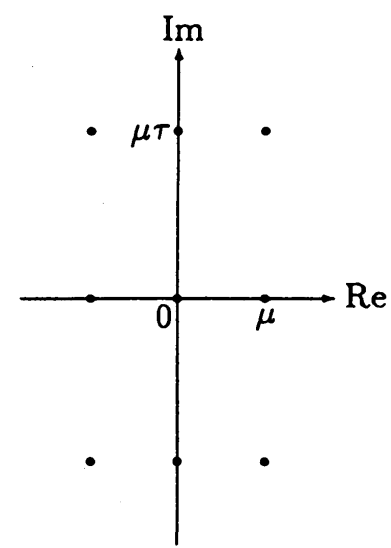

(i)

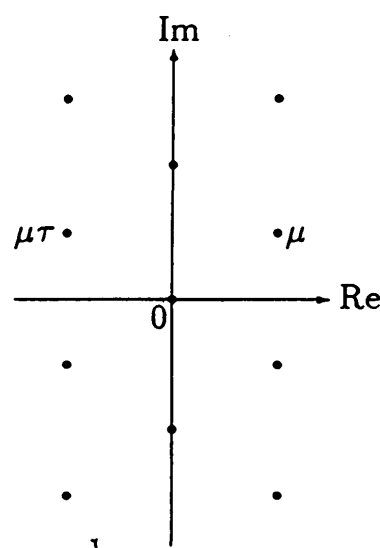

(ii)

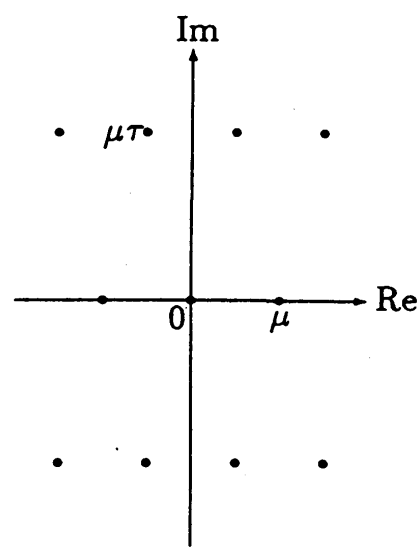

(iii)

Lattice points are represented by $\bullet$. (i) The case $\tau \in L_{1}$ and $\mu>0$. (ii) The case $\tau \in L_{2}$ and $\mu=$ $r e^{(\pi-\theta) i / 2}$ with $r>0$. (iii) The case $\tau \in L_{3}$ and $\mu>0$.

Figure 2.1
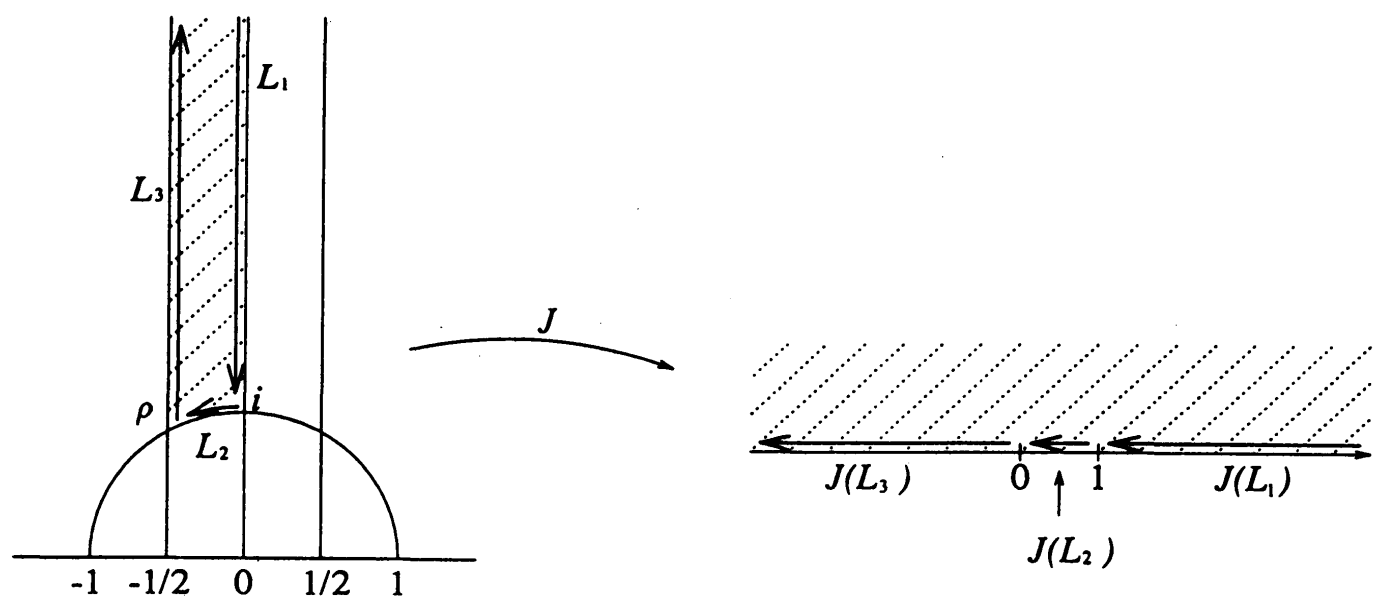

FIGURE 2.2 
(ii) The mapping $J: \mathbf{H} \rightarrow \mathbf{C}$ is holomorphic on $\mathbf{H}$.

(iii) $J$ maps $L$ onto $\mathbf{R}$ where $L=L_{1} \cup L_{2} \cup L_{3}$. In particular, $J(i)=1$ and $J(\rho)=0$. (See Fig. 2.2.)

(iv) J maps $F$ onto $\mathbf{C}$ where $F=\{\tau \in \mathbf{H}|| \tau \mid \geq 1$ and $|\operatorname{Re}(\tau)| \leq 1 / 2\}$ is a fundamental domain for the modular group.

By using $g_{2}$ and $g_{3}$, we can introduce an important differential equation connecting $\wp(u)$ and $\wp^{\prime}(u)$ :

PROPOSITION 2.4. $\wp^{\prime}(u)^{2}=4 \wp(u)^{3}-g_{2 \wp}(u)-g_{3}$.

Note that $\wp, g_{2}$ and $g_{3}$ depend on a lattice $\Omega$.

PROPOSITION 2.5. Let $\Omega=\Omega\left(\omega_{1}, \omega_{2}\right)$ and $\omega_{3}=\omega_{1}+\omega_{2}$. Then we have $\wp^{\prime}\left(\omega_{1} / 2\right)=$ $\wp^{\prime}\left(\omega_{2} / 2\right)=\wp^{\prime}\left(\omega_{3} / 2\right)=0$

Points $\omega_{k} / 2+\left(n_{1} \omega_{1}+n_{2} \omega_{2}\right)$ for all $n_{1}, n_{2} \in \mathbf{Z}$ and $k=1,2,3$ are called ramification points of $\Omega$.

Proposition 2.6. We define $e_{k}=\wp\left(\omega_{k} / 2\right)$ for $k=1,2,3$. Then $e_{1}, e_{2}, e_{3}$ are mutually distinct.

That is to say, if we take a lattice $\Omega$, the values $g_{2}(\Omega)$ and $g_{3}(\Omega)$ define a cubic polynomial $p(x)=4 x^{3}-g_{2} x-g_{3}$, which has distinct roots. Conversely, we obtain the following assertion.

Proposition 2.7. Let $p(x)=4 x^{3}-c_{2} x-c_{3}$ for $c_{2}, c_{3} \in \mathbf{C}$ be any cubic polynomial with distinct roots. Then there is a lattice $\Omega$ with $c_{2}=g_{2}(\Omega)$ and $c_{3}=g_{3}(\Omega)$. More precisely, $=c_{3}$.

(i) if $c_{2}=0, c_{3} \neq 0$ then $\Omega=\Omega(\mu, \mu \rho)$, where $\mu \in \mathbf{C}$ is determined by $\left(1 / \mu^{6}\right) g_{3}(\rho)$

(ii) if $c_{2} \neq 0, c_{3}=0$ then $\Omega=\Omega(\mu, \mu i)$, where $\mu \in \mathbf{C}$ is determined by $\left(1 / \mu^{4}\right) g_{2}(i)$ $=c_{2}$.

(iii) if $c_{2} \neq 0, c_{3} \neq 0$ then $\Omega=\Omega(\mu, \mu \tau)$, where $\mu$ is any element of $\mathbf{C} \backslash\{0\}$ and $\tau \in \mathbf{C}$ is determined by $J(\tau)=c_{2}^{3} /\left(c_{2}^{3}-27 c_{3}^{2}\right)$.

We introduce two notations: one is $\mu \mathbf{R}=\{\mu u \mid u \in \mathbf{R}\}$ for $\mu \in \mathbf{C}$ and the other is $\wp\left(\mu_{1} \mathbf{R}\right) \subset \mu_{2} \mathbf{R} \cup\{\infty\}$ for $\mu_{1}, \mu_{2} \in \mathbf{C}$ which means $\wp\left(\mu_{1} u\right) \in \mu_{2} \mathbf{R} \cup\{\infty\}$ for all $u \in \mathbf{R}$.

Proposition 2.8. (i) If $\Omega$ is a real lattice, then $\wp(\mathbf{R}) \subset \mathbf{R} \cup\{\infty\}$ and $\wp(i \mathbf{R}) \subset$ $\mathbf{R} \cup\{\infty\}$

(ii) If $\Omega=\Omega(\mu, \mu \rho)$ for some $\mu \in \mathbf{C}$ is a real lattice, then

$$
\begin{array}{ll}
\wp(\rho \mathbf{R}) \subset \rho \mathbf{R} \cup\{\infty\}, & \wp\left(\rho^{1 / 2} \mathbf{R}\right) \subset \rho^{1 / 2} \mathbf{R} \cup\{\infty\}, \\
\wp(\rho i \mathbf{R}) \subset \rho \mathbf{R} \cup\{\infty\}, & \wp\left(\rho^{1 / 2} i \mathbf{R}\right) \subset \rho^{1 / 2} \mathbf{R} \cup\{\infty\} .
\end{array}
$$


(iii) If $\Omega=\Omega(\mu, \mu i)$ for some $\mu \in \mathbf{C}$ is a real lattice, then

$$
\wp\left(e^{\pi i / 4} \mathbf{R}\right) \subset i \mathbf{R} \cup\{\infty\}, \quad \wp\left(e^{-\pi i / 4} \mathbf{R}\right) \subset i \mathbf{R} \cup\{\infty\} .
$$

Proof. (i) By using Proposition 2.2 (ii), $\wp$ satisfies $\wp(u)=\wp(\bar{u})=\overline{\wp(u)}$ for all $u \in \mathbf{R}$ which means $\wp(\mathbf{R}) \subset \mathbf{R} \cup\{\infty\}$. For all $u \in i \mathbf{R}$ we have $\wp(u)=\wp(-u)=\wp(\bar{u})=$ $\overline{\wp(u)}$. Therefore $\wp(i \mathbf{R}) \subset \mathbf{R} \cup\{\infty\}$.

(ii) For $\rho=e^{2 \pi i / 3}$ we easily have

$$
\rho \Omega(\mu, \mu \rho)=\rho^{1 / 2} \Omega(\mu, \mu \rho)=\Omega(\mu, \mu \rho) .
$$

By using these relations, we obtain

$$
\wp(\rho u)=\frac{1}{\rho^{2}} \wp(u) \quad \text { and } \quad \wp\left(\rho^{1 / 2} u\right)=\frac{1}{\rho} \wp(u) \quad \text { for all } u \in \mathbf{C} .
$$

Taking $u \in \mathbf{R}$ or $u \in i \mathbf{R}$ and using (i) of this proposition, we get the assertions.

(iii) From the fact

$$
i \Omega(\mu, \mu i)=-i \Omega(\mu, \mu i)=\Omega(\mu, \mu i),
$$

we have

$$
\wp(i u)=\wp(-i u)=-\wp(u) \text { for all } u \in \mathbf{C} .
$$

If we take $u=t e^{\pi i / 4}, t \in \mathbf{R}$, then $\bar{u}=t e^{-\pi i / 4}=-i u$. As $\Omega(\mu, \mu i)$ is a real lattice, we can use Proposition 2.2 (ii) and have

$$
\overline{\wp(u)}=\wp(\bar{u})=\wp(-i u)=-\wp(u) .
$$

Therefore $\wp(u)$ is purely imaginary, i.e., $\wp\left(e^{\pi i / 4} \mathbf{R}\right) \subset i \mathbf{R} \cup\{\infty\}$. From this result and (2.1) we obtain $\wp\left(e^{-\pi i / 4} \mathbf{R}\right) \subset i \mathbf{R} \cup\{\infty\}$.

By using the same arguments as in the proof of Proposition 2.8 we obtain the following assertions.

Proposition 2.9. For the lattice $\Omega=\Omega(v, v \rho)$ with $v=r e^{\pi i / 6}$ for some $r>0$, we have

$$
\begin{array}{crl}
\wp((v+\rho v) / 2+\mathbf{R}) \subset \mathbf{R} \cup\{\infty\}, & \wp(-(\nu+\rho \nu) / 2+\mathbf{R}) \subset \mathbf{R} \cup\{\infty\}, \\
\wp(\rho((\nu+\rho v) / 2+\mathbf{R})) \subset \rho \mathbf{R} \cup\{\infty\}, & \wp(\rho(-(v+\rho \nu) / 2+\mathbf{R})) \subset \rho \mathbf{R} \cup\{\infty\}, \\
\wp\left(\rho^{1 / 2}((\nu+\rho v) / 2+\mathbf{R})\right) \subset \rho^{1 / 2} \mathbf{R} \cup\{\infty\}, & \wp\left(\rho^{1 / 2}(-(\nu+\rho \nu) / 2+\mathbf{R})\right) \subset \rho^{1 / 2} \mathbf{R} \cup\{\infty\} .
\end{array}
$$

Proposition 2.10. For the lattice $\Omega=\Omega(\nu, v i)$ with $v>0$, we have

$$
\begin{array}{ll}
\wp(\mathbf{R}+v i / 2) \subset \mathbf{R} \cup\{\infty\}, & \wp(\mathbf{R}-v i / 2) \subset \mathbf{R} \cup\{\infty\}, \\
\wp(i \mathbf{R}+v / 2) \subset \mathbf{R} \cup\{\infty\}, & \wp(i \mathbf{R}-v / 2) \subset \mathbf{R} \cup\{\infty\} .
\end{array}
$$

\section{Correspondences as sets.}

In this section we will introduce the Teichmüller modular group for $\mathcal{T}_{1,1}$ and will characterize subsets of a fundamental domain for the action of the modular group on $\mathcal{T}_{1,1}$. Recall 
that the Teichmüller modular group for $\mathcal{T}_{1,0}$ is $\operatorname{SL}(2, \mathbf{Z})$ and a fundamental domain for the action of $\operatorname{PSL}(2, \mathbf{Z})$ on $\mathcal{T}_{1,0}$ is the region $F$ introduced in Proposition 2.3 (iv).

Let $G$ be the free group with generators $A$ and $B$. The automorphism group $\operatorname{Aut}(G)$ of $G$ has generators $\sigma, P$ and $U$ which are defined by

$$
\begin{array}{cl}
\sigma(A)=A^{-1}, & \sigma(B)=B, \\
P(A)=B, & P(B)=A, \\
U(A)=A B, & U(B)=B .
\end{array}
$$

A representation of $\operatorname{Aut}(G)$ in $\operatorname{GL}(2, \mathbf{Z})$ is obtained by

$$
\sigma \rightarrow\left(\begin{array}{cc}
-1 & 0 \\
0 & 1
\end{array}\right), \quad P \rightarrow\left(\begin{array}{ll}
0 & 1 \\
1 & 0
\end{array}\right) \quad \text { and } U \rightarrow\left(\begin{array}{ll}
1 & 1 \\
0 & 1
\end{array}\right)
$$

Let $\operatorname{Aut}^{+}(G)$ be the preimage of $\operatorname{SL}(2, \mathbf{Z})$ and let $\operatorname{Inn}(G)$ be the inner automorphism group of $G$. Then we have the following theorem.

THEOREM 3.1 (Nielsen). Let $G$ be the free group with two generators. Then

$$
\operatorname{Out}^{+}(G)=\operatorname{Aut}^{+}(G) / \operatorname{Inn}(G) \approx \operatorname{SL}(2, \mathbf{Z}) .
$$

The proof can be found in [MKS].

Therefore the Teichmüller modular group $\mathcal{M}$ for $\mathcal{T}_{1,1}$ is $\mathrm{Out}^{+}(G)$. The kernel of the action of the modular group $\mathcal{M}$ on $\mathcal{T}_{1,1}$ is $\{ \pm I\}$, where $I=\left(\begin{array}{ll}1 & 0 \\ 0 & 1\end{array}\right)$, so $\mathcal{P} \mathcal{M}=\operatorname{Out}^{+}(G) /\{ \pm I\} \approx$ $\operatorname{PSL}(2, \mathbf{Z})$ acts effectively on $\mathcal{T}_{1,1}$.

THEOREM 3.2. A fundamental domain for the action of $\mathcal{P} \mathcal{M}$ on $\mathcal{T}_{1,1}$ is

$$
M=\left\{(X, Y, Z) \in \mathcal{T}_{1,1} \mid 2<X \leq Y \leq Z \leq \frac{1}{2} X Y\right\}
$$

Before we give a proof of Theorem 3.2, we prepare some facts. The generators $\sigma, P$ and $U$ induce actions on the $(X, Y, Z)$ coordinates, where $X=\operatorname{tr} A, Y=\operatorname{tr} B$ and $Z=\operatorname{tr} A B$ :

$$
\begin{array}{ccc}
\sigma(X)=X, & \sigma(Y)=Y, & \sigma(Z)=X Y-Z, \\
P(X)=Y, & P(Y)=X, & P(Z)=Z, \\
U(X)=Z, & U(Y)=Y, & U(Z)=Y Z-X .
\end{array}
$$

In order to introduce these actions we used the following properties: $\operatorname{tr} C^{-1}=\operatorname{tr} C, \operatorname{tr} C D=$ $\operatorname{tr} D C$ and $\operatorname{tr} C \operatorname{tr} D=\operatorname{tr} C D+\operatorname{tr} C^{-1} D$ for all $C, D \in \operatorname{PSL}(2, \mathbf{R})$.

LEMMA 3.1. The actions of $\sigma, P$ and $U$ on a point $(X, Y, Z) \in \mathcal{T}_{1,1}$

$$
\begin{gathered}
\sigma(X, Y, Z)=(X, Y, X Y-Z), \\
P(X, Y, Z)=(Y, X, Z), \\
U(X, Y, Z)=(Z, Y, Y Z-X)
\end{gathered}
$$

are well-defined. 
Proof. We consider the action of $\sigma$. The equation $X^{2}+Y^{2}+(X Y-Z)^{2}=$ $X Y(X Y-Z)$ is equal to $X^{2}+Y^{2}+Z^{2}=X Y Z$. We easily get $X Y-Z=$ $\left(X Y \mp \sqrt{X^{2} Y^{2}-4\left(X^{2}+Y^{2}\right)}\right) / 2>2$. By using the same calculation, the action of $U$ is well-defined. It is clear that the action of $P$ is well-defined.

Next we introduce another coordinate system $[a, b, c]$ for $\mathcal{T}_{1,1}$ by

$$
a=\frac{X}{Y Z}, \quad b=\frac{Y}{X Z} \quad \text { and } \quad c=\frac{Z}{X Y} .
$$

Then the space $\mathcal{T}_{1,1}$ and the set $M$ are represented by

$$
\begin{aligned}
\mathcal{T}_{1,1} & =\{[a, b, c] \mid a+b+c=1 \text { with } a, b, c>0\}, \\
M & =\left\{[a, b, c] \in \mathcal{T}_{1,1} \mid 0<a \leq b \leq c \leq \frac{1}{2}\right\} .
\end{aligned}
$$

Note that we use $(\cdot)$ for the $(X, Y, Z)$ coordinates and $[\cdot]$ for the $[a, b, c]$ coordinates and that the space $\mathcal{T}_{1,1}$ represented by $[a, b, c]$ (Fig. 1.1) is much easier to visualize than by $(X, Y, Z)$. We also have actions of $\sigma, P$ and $U$ in the $[a, b, c]$ coordinates:

$$
\begin{gathered}
\sigma[a, b, c]=\left[\frac{a c}{1-c}, \frac{b c}{1-c}, 1-c\right], \\
P[a, b, c]=[b, a, c], \\
U[a, b, c]=\left[\frac{a c}{1-a}, \frac{a b}{1-a}, 1-a\right] .
\end{gathered}
$$

From definitions we can easily get the following facts.

LEMMA 3.2. There exist actions which permute coordinates $(X, Y, Z)$ and $[a, b, c]$.

$$
\begin{array}{cc}
\sigma U(X, Y, Z)=(Z, Y, X), & P \sigma U(X, Y, Z)=(Y, Z, X), \\
\sigma U P(X, Y, Z)=(Z, X, Y), & \sigma U P \sigma U(X, Y, Z)=(X, Z, Y), \\
\sigma U[a, b, c]=[c, b, a], & P \sigma U[a, b, c]=[b, c, a], \\
\sigma U P[a, b, c]=[c, a, b], & \sigma U P \sigma U[a, b, c]=[a, c, b] .
\end{array}
$$

Proof of THEOREM 3.2. We use the following results from [W]. Let $\mathcal{M}_{2}$ be the subgroup of $\operatorname{Aut}(G)$ generated by $\rho_{1}=\sigma U^{2}, \rho_{2}=\sigma$ and $\rho_{3}=P \sigma U^{2} P$. A fundamental domain for the action of $\mathcal{M}_{2}$ on $\mathcal{T}_{1,1}$ is

$$
\tilde{M}=\left\{[a, b, c] \in \mathcal{T}_{1,1} \mid a, b, c \leq \frac{1}{2}\right\} .
$$

Set $\mathcal{M}_{2}^{+}=\mathcal{M}_{2} \cap$ Aut $^{+}(G)$. The group $\mathcal{M}_{2}^{+}$acts effectively on $\mathcal{T}_{1,1}$ and the index $\operatorname{PSL}(2, \mathbf{Z})$ : $\left.\mathcal{M}_{2}^{+}\right]=6$.

Recall that $\mathcal{P M} \approx \operatorname{PSL}(2, \mathbf{Z})$. We can obtain the following coset representation: if we set

$$
\left\{\alpha_{0}, \alpha_{1}, \alpha_{2}, \alpha_{3}, \alpha_{4}, \alpha_{5}\right\}=\{i d, P, \sigma U, P \sigma U, \sigma U P, \sigma U P \sigma U\}
$$

then $\tilde{M}=\bigcup_{i=0}^{5} \alpha_{i} M, \alpha_{i} M^{0} \cap \alpha_{j} M^{0}=\emptyset$ for $i, j \in\{0,1,2,3,4,5\}, i \neq j$, where $M^{0}$ is the interior of $M$. 
Since the Teichmüller space $\mathcal{T}_{1,0}$ is the upper half-plane $\mathbf{H}$ and the Teichmüller modular group for $\mathcal{T}_{1,0}$ is $\operatorname{SL}(2, \mathbf{Z})$, the region $F$ in Proposition 2.3 is a fundamental domain for the action of $\operatorname{PSL}(2, \mathbf{Z})$ on $\mathcal{T}_{1,0}$ in the sense of the Teichmüller theory. Therefore it is natural that we study a correspondence between the fundamental domain $M$ in $\mathcal{T}_{1,1}$ and the fundamental domain $F$ in $\mathcal{T}_{1,0}$.

We introduce some specific subsets of the fundamental domain $M$. We consider the automorphisms $\Phi_{1}(A, B)=\left(A, B^{-1}\right)$ and $\Phi_{1}^{\prime}(A, B)=\left(A^{-1}, B\right)$. The induced actions on $\mathcal{T}_{1,1}$ are described by

$$
\Phi_{1}(X, Y, Z)=\Phi_{1}^{\prime}(X, Y, Z)=(X, Y, X Y-Z) .
$$

Let $M_{1}$ be the subset of $M$ whose point is fixed by $\Phi_{1}$ and $\Phi_{1}^{\prime}$. From $X Y-Z=Z$ we have

$$
M_{1}=\left\{(X, Y, Z) \in M \mid Z=\frac{1}{2} X Y\right\}=\left\{[a, b, c] \in M \mid c=\frac{1}{2}\right\} .
$$

In the same way we consider the automorphisms:

$$
\begin{gathered}
\Phi_{2}\left(A, B^{-1}\right)=\left(B^{-1}, A\right), \quad \Phi_{2}^{\prime}(A, B)=(B, A), \\
\Phi_{3}(A, B)=\left(A, A^{-1} B^{-1}\right), \quad \Phi_{3}^{\prime}(A, B)=\left(A^{-1}, A B\right) .
\end{gathered}
$$

Then natural induced actions on $\mathcal{T}_{1,1}$ are as follows:

$$
\begin{aligned}
& \Phi_{2}(X, Y, Z)=\Phi_{2}^{\prime}(X, Y, Z)=(Y, X, Z), \\
& \Phi_{3}(X, Y, Z)=\Phi_{3}^{\prime}(X, Y, Z)=(X, Z, Y) .
\end{aligned}
$$

We define subsets $M_{k}$ as sets whose point is fixed by $\Phi_{k}$ and $\Phi_{k}^{\prime}, k=2,3$ :

$$
\begin{aligned}
& M_{2}=\{(X, Y, Z) \in M \mid X=Y\}=\{[a, b, c] \in M \mid a=b\}, \\
& M_{3}=\{(X, Y, Z) \in M \mid Y=Z\}=\{[a, b, c] \in M \mid b=c\} .
\end{aligned}
$$

THEOREM 3.3. We can establish the correspondences among the sets as follows:

$$
L_{1} \Leftrightarrow M_{1}, \quad L_{2} \Leftrightarrow M_{2}, \quad L_{3} \Leftrightarrow M_{3} .
$$

Proof. We take a real lattice $\Omega=\Omega(\mu, \mu \tau)$ defined in $\S 2$. Let $\Gamma=\langle A, B\rangle$ be a Fricke group and let $(\tilde{X}, \tilde{Y}, \tilde{Z})$ be the coordinate of $\Gamma$ in $\mathcal{T}_{1,1}$. We define correspondences between the basis $(\mu, \mu \tau)$ of $\Omega$ and the generators $A, B$ of $\Gamma$ as follows:

$$
\mu \Leftrightarrow A \text { and } \mu \tau \Leftrightarrow B \text {. }
$$

The general real lattice has symmetry with respect to the real axis and the imaginary axis and does not have symmetry with respect to any other line through the origin except the real and the imaginary axes. The only exceptions are $\Omega=\Omega(\mu, \mu \tau)$ for $\tau=i$ or $\rho=e^{2 \pi i / 3}$. Conversely, a lattice which is symmetric with respect to the real and the imaginary axes is a real lattice.

If $\tau \in L_{1}$, we consider a rectangular lattice $\Omega=\Omega(\mu, \mu \tau)$ for $\mu>0$ and define transformations of the basis of the lattice $\Omega$ as

$$
\varphi_{1}(\mu, \mu \tau)=(\mu,-\mu \tau) \quad \text { and } \quad \varphi_{1}^{\prime}(\mu, \mu \tau)=(-\mu, \mu \tau) .
$$


The symmetry of $\Omega$ with respect to the real axis is characterized by $\varphi_{1}(\Omega)=\Omega$ and the symmetry of $\Omega$ with respect to the imaginary axis is characterized by $\varphi_{1}^{\prime}(\Omega)=\Omega$. By the correspondences (3.1) the transformations $\varphi_{1}$ and $\varphi_{1}^{\prime}$ correspond to the automorphisms $\Phi_{1}$ and $\Phi_{1}^{\prime}$, respectively. Then the equation $\varphi_{1}(\Omega)=\varphi_{1}^{\prime}(\Omega)=\Omega$ is equivalent to $\Phi_{1}(\tilde{X}, \tilde{Y}, \tilde{Z})=$ $\Phi_{1}^{\prime}(\tilde{X}, \tilde{Y}, \tilde{Z})=(\tilde{X}, \tilde{Y}, \tilde{Z})$. Therefore we get $(\tilde{X}, \tilde{Y}, \tilde{Z}) \in M_{1}$ corresponding to $\tau \in L_{1}$.

If $\tau \in L_{2}$, we take a rhombic lattice $\Omega=\Omega(\mu, \mu \tau)$, where $\tau=e^{i \theta}, \mu=r e^{(\pi-\theta) i / 2}$, $r>0$ and define transformations of the basis of the lattice $\Omega$ as

$$
\varphi_{2}(\mu,-\mu \tau)=(-\mu \tau, \mu) \text { and } \varphi_{2}^{\prime}(\mu, \mu \tau)=(\mu \tau, \mu) .
$$

If $\tau \in L_{3}$, we consider transformations of the basis of a rhombic lattice $\Omega=\Omega(\mu, \mu \tau)$ for $\mu>0$ :

$$
\varphi_{3}(\mu, \mu \tau)=(\mu, \overline{\mu \tau}) \quad \text { and } \quad \varphi_{3}^{\prime}(\mu, \mu \tau)=(-\mu,-\overline{\mu \tau})
$$

By using the same argument as for the case $\tau \in L_{1}$ we obtain the assertions.

COROLlaRY 3.1. The points $i, \rho \in \mathcal{T}_{1,0}$ correspond to $(2 \sqrt{2}, 2 \sqrt{2}, 4),(3,3,3) \in$ $\mathcal{T}_{1,1}$, respectively.

Proof. Since the symmetries of $\Omega=\Omega(\mu, \mu i)$ for $\mu>0$ are characterized by $\varphi_{1}(\Omega)=\varphi_{1}^{\prime}(\Omega)=\varphi_{2}(\Omega)=\varphi_{2}^{\prime}(\Omega)=\Omega$, the point $(\tilde{X}, \tilde{Y}, \tilde{Z})$ corresponding to $i$ must satisfy $\Phi_{1}(\tilde{X}, \tilde{Y}, \tilde{Z})=\Phi_{1}^{\prime}(\tilde{X}, \tilde{Y}, \tilde{Z})=\Phi_{2}(\tilde{X}, \tilde{Y}, \tilde{Z})=\Phi_{2}^{\prime}(\tilde{X}, \tilde{Y}, \tilde{Z})=(\tilde{X}, \tilde{Y}, \tilde{Z})$. Then it is $(2 \sqrt{2}, 2 \sqrt{2}, 4)$. In the same way we obtain another correspondence.

\section{Two examples.}

Recall that we represent a point in the upper half-plane $\mathbf{H}$ by $z$ and a point in the complex plane $\mathbf{C}$ by $u$ and that we call $\mathbf{H}$ the $z$-plane and $\mathbf{C}$ the $u$-plane. Let $\Gamma$ be a Fricke group associated with a once punctured torus $(X, Y, Z) \in \mathcal{T}_{1,1}$. Then $\Gamma$ determines a fundamental domain in the $z$-plane, which can be identified with the once punctured torus $(X, Y, Z)$. Let $\Gamma_{\tau}$ be a lattice in the $u$-plane for $\tau \in \mathcal{T}_{1,0}$. Then a fundamental domain for $\Gamma_{\tau}$ in the $u$-plane can be identified with the closed torus $\tau$.

In this section we consider two points $(3,3,3),(2 \sqrt{2}, 2 \sqrt{2}, 4)$ in $\mathcal{T}_{1,1}$. We know that they must correspond to $\rho, i \in \mathcal{T}_{1,0}$ (Corollary 3.1). Before we show a construction of a holomorphic mapping between a once punctured torus and a closed torus, we display fundamental domains in the $z$-plane for Fricke groups associated with these points.

(I) the case $(3,3,3)$.

Let $\Gamma_{\rho}=\left\langle A_{\rho}, B_{\rho}\right\rangle$ with $A_{\rho}=\left(\begin{array}{ll}1 & 1 \\ 1 & 2\end{array}\right)$ and $B_{\rho}=\left(\begin{array}{cc}1 & -1 \\ -1 & 2\end{array}\right)$ be a representation of $(3,3,3)$. Define $C_{\rho}=B_{\rho}^{-1} A_{\rho}^{-1}$. Then $C_{\rho}=\left(\begin{array}{cc}3 & -1 \\ 1 & 0\end{array}\right)$ and $C_{\rho} B_{\rho} A_{\rho}=\left(\begin{array}{cc}-1 & -6 \\ 0 & -1\end{array}\right)$. We can obtain two fundamental domains: one is a quadrilateral whose opposite sides are identified by actions of $A_{\rho}$ and $B_{\rho}$ (shown in normal outline in Fig. 4.1),

$$
D_{q}\left(\Gamma_{\rho}\right)=\left\{z \in \mathbf{H}|| z+\frac{1}{2}\left|>\frac{1}{2},\right| z-\frac{1}{2} \mid>\frac{1}{2},-1 \leq \operatorname{Re}(z) \leq 1\right\} \text {, }
$$




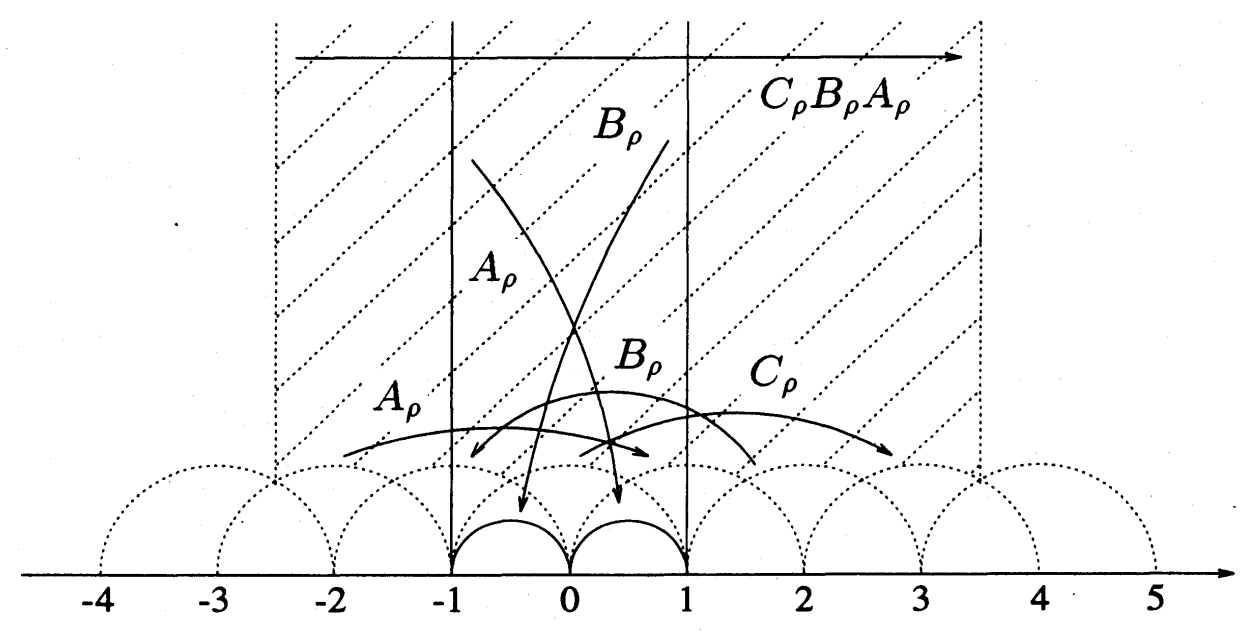

FIGURE 4.1

the other is an octagon whose sides are identified by actions of $A_{\rho}, B_{\rho}, C_{\rho}$ and $C_{\rho} B_{\rho} A_{\rho}$

$$
\begin{aligned}
D_{o}\left(\Gamma_{\rho}\right)= & \{z \in \mathbf{H}|| z+2|\geq 1,| z+1|\geq 1,| z \mid \geq 1, \\
& \left.|z-1|>1,|z-2|>1,|z-3|>1,-\frac{5}{2} \leq \operatorname{Re}(z)<\frac{7}{2}\right\},
\end{aligned}
$$

(the shaded part in Fig. 4.1). We call the former the quadrilateral fundamental domain and the latter the octagonal fundamental domain.

(II) the case $(2 \sqrt{2}, 2 \sqrt{2}, 4)$.

As a representation of $(2 \sqrt{2}, 2 \sqrt{2}, 4)$ we take $\Gamma_{i}=\left\langle A_{i}, B_{i}\right\rangle$ with $A_{i}=\left(\begin{array}{cc}0 & -1 \\ 1 & 2 \sqrt{2}\end{array}\right)$ and $B_{i}=\left(\begin{array}{cc}\sqrt{2} & -1 \\ -1 & \sqrt{2}\end{array}\right)$ and define $C_{i}=B_{i}^{-1} A_{i}^{-1}$. Then $C_{i} B_{i} A_{i}=\left(\begin{array}{cc}-1 & -4 \sqrt{2} \\ 0 & -1\end{array}\right)$. One fundamental domain is a quadrilateral whose opposite sides are identified by actions of $A_{i}$ and $B_{i}$ (shown in normal outline in Fig. 4.2),

$$
D_{q}\left(\Gamma_{i}\right)=\left\{z \in \mathbf{H}|| z+\frac{3 \sqrt{2}}{4}\left|>\frac{\sqrt{2}}{4},\right| z+\frac{\sqrt{2}}{4} \mid>\frac{\sqrt{2}}{4},-\sqrt{2} \leq \operatorname{Re}(z) \leq 0\right\},
$$

another is a hexagon whose sides are identified by actions of $A_{i}, B_{i}$ and $C_{i} B_{i} A_{i}$ (the shaded part in Fig. 4.2),

$$
\begin{aligned}
D_{h}\left(\Gamma_{i}\right)= & \{z \in \mathbf{H}|| z+2 \sqrt{2}|\geq 1,| z+\sqrt{2} \mid \geq 1, \\
& \left.|z|>1,|z-\sqrt{2}|>1,-\frac{5 \sqrt{2}}{2} \leq \operatorname{Re}(z)<\frac{3 \sqrt{2}}{2}\right\} .
\end{aligned}
$$




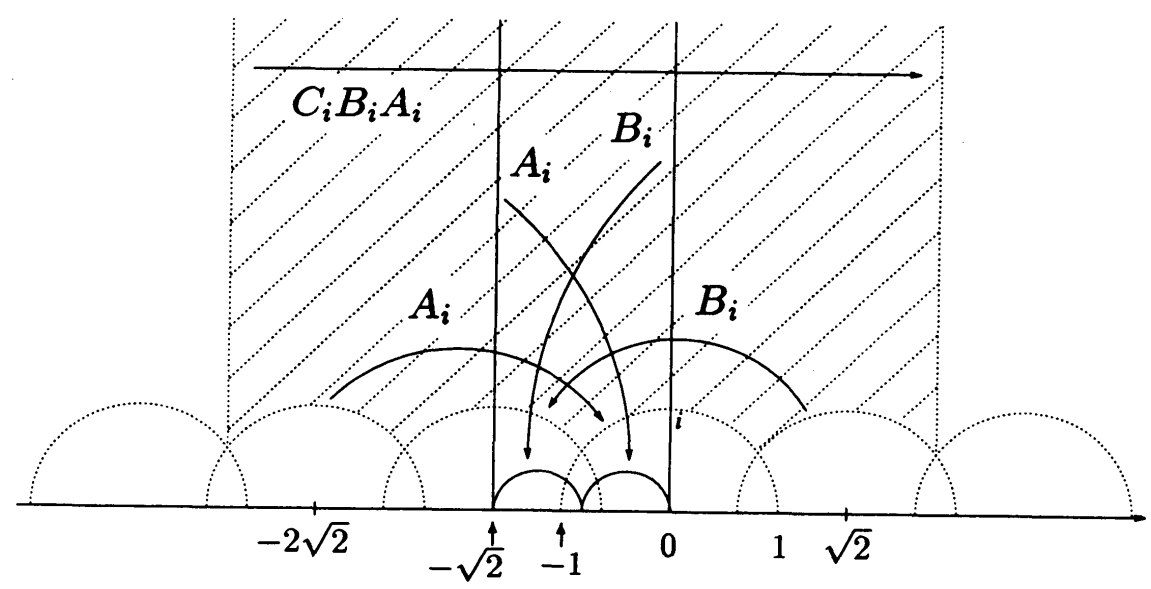

FIGURE 4.2

THEOREM 4.1 (Cohn). The once punctured torus $(3,3,3) \in \mathcal{T}_{1,1}$ is mapped to the closed torus $\rho=e^{2 \pi i / 3} \in \mathcal{T}_{1,0}$ holomorphically by using the relation

$$
1-J(z)=\wp^{\prime}(u)^{2}=4 \wp(u)^{3}+1 .
$$

Proof. We take $\Gamma_{\rho}=\left\langle A_{\rho}, B_{\rho}\right\rangle$ with $A_{\rho}=\left(\begin{array}{ll}1 & 1 \\ 1 & 2\end{array}\right)$ and $B_{\rho}=\left(\begin{array}{cc}1 & -1 \\ -1 & 2\end{array}\right)$ as a representation of $(3,3,3)$, then two fundamental domains for $\Gamma_{\rho}$ in the $z$-plane are as shown in Fig. 4.1. By Proposition 2.7 (i), the relation

$$
\wp^{\prime}(u)^{2}=4 \wp(u)^{3}+1
$$

determines the lattice $\Omega=\Omega(\mu, \mu \rho)$ in the $u$-plane where $\mu$ satisfies $\left(1 / \mu^{6}\right) g_{3}(\rho)=-1$. As $g_{3}(\rho)>0$, we can write $\mu=|\mu| e^{(1 / 6+j / 3) \pi i}, j=0,1,2,3,4,5$. Therefore we obtain $\Omega=\Omega(\mu, \mu \rho)=\Omega\left(|\mu| e^{\pi i / 6},|\mu| e^{\pi i / 6} \rho\right)$ which is a real lattice. By the relation

$$
1-J(z)=4 \wp(u)^{3}+1,
$$

the point $z=i \infty$ is mapped to a lattice point of the $u$-plane because of $J(i \infty)=\infty$ and $\wp\left(u_{0}\right)=\infty$ for $u_{0} \in \Omega=\Omega(\mu, \mu \rho)$. We can assume without loss of generality that the image of $z=i \infty$ by (4.3) is the point $u=0$. Suppose that $J$ has a value $c_{0} \in \mathbf{C}$ with $c_{0} \neq 1$. Since for each $c \in \mathbf{C}, c \neq e_{1}, e_{2}, e_{3}, \infty$ the equation $\wp(u)=c$ has two simple solutions, for $J=c_{0}$ we obtain six values of $u$ determined by the relation (4.3). The octagonal fundamental domain for $\Gamma$ is a 6-sheeted covering of the fundamental domain $F$ for the modular group $\operatorname{PSL}(2, \mathbf{Z})$, so we can take six points which attain $J=c_{0}$ in the octagonal fundamental domain. Therefore we can obtain correspondences between these six points in the $z$-plane and the six points in the $u$-plane. Now we consider the case where $J(z)$ is real. From Proposition 2.3 (iii) we divide arguments into the following three cases.

(I) $J(z) \geq 1$, i.e., $z \in L_{1}$.

Set $J(z)=c$ then by using (4.3) we have 


$$
\wp(u)=\left\{\begin{array}{l}
-(c / 4)^{1 / 3}, \\
(c / 4)^{1 / 3} e^{\pi i / 3}, \\
(c / 4)^{1 / 3} e^{-\pi i / 3} .
\end{array}\right.
$$

By Proposition 2.8 (i) we have $\wp(i \mathbf{R}) \subset \mathbf{R} \cup\{\infty\}$ and if we write $u=i t$ and $t \in \mathbf{R}$ is small, $\wp(u)$ is negative and $|\wp(u)|$ is large, so we can get a point $u$ with $\wp(u)=-(c / 4)^{1 / 3}$ on the line $i \mathbf{R}$. By using the same argument we can get a point $u$ with $\wp(u)=(c / 4)^{1 / 3} e^{\pi i / 3}$ on $\rho^{1 / 2} i \mathbf{R}$ and a point $u$ with $\wp(u)=(c / 4)^{1 / 3} e^{-\pi i / 3}$ on $\rho i \mathbf{R}$. If $J(i)=1$ in (4.3) then $4 \wp^{3}(u)+1=0$. From (4.2) the point $u$ corresponding to $z=i$ must be ramification points $\frac{1}{2}|\mu| e^{(1 / 6+j / 3) \pi i}, j=0,1,2,3,4,5$ in the $u$-plane. Therefore we can take segments $\left\{t|\mu| e^{(1 / 6+j / 3) \pi i} \mid 0 \leq t \leq \frac{1}{2}\right\}, j=0,1,2,3,4,5$ as images of $L_{1}$ by the mapping defined by the relation (4.1).

(II) $J(z) \leq 0$, i.e., $z \in L_{3}$.

Set $J(z)=c$ then by using (4.3) we have

$$
\wp(u)=\left\{\begin{array}{l}
(-c / 4)^{1 / 3}, \\
(-c / 4)^{1 / 3} e^{2 \pi i / 3}, \\
(-c / 4)^{1 / 3} e^{-2 \pi i / 3} .
\end{array}\right.
$$

By Proposition 2.8 (i) we have $\wp(\mathbf{R}) \subset \mathbf{R} \cup\{\infty\}$ and if $u=t \in \mathbf{R}$ and $t$ is small, $\wp(u)$ is positively large, so we can take a point $u$ with $\wp(u)=(-c / 4)^{1 / 3}$ on the line $\mathbf{R}$. In the same way we can take a point $u$ with $\wp(u)=(-c / 4)^{1 / 3} e^{2 \pi i / 3}$ on $\rho \mathbf{R}$ and a point $u$ with $\wp(u)=(-c / 4)^{1 / 3} e^{-2 \pi i / 3}$ on $\rho^{1 / 2} \mathbf{R}$.

(III) $0 \leq J(z) \leq 1$, i.e., $z \in L_{2}$.

By setting $J(z)=c, \wp(u)$ satisfies (4.4). Since $J(z)$ is holomorphic on $\mathbf{H}$ and $\wp(u)$ is holomorphic on $\mathbf{C} / \Omega$, the mapping defined by the relation (4.1) is conformal on $\mathbf{C} / \Omega$. Therefore we obtain Fig. 4.3 (i) by Proposition 2.9 and the arguments in (I) and (II).

From Proposition 2.3 and the symmetry of the fundamental domain $F$, the image of the octagonal fundamental domain $D_{o}\left(\Gamma_{\rho}\right)$ by the mapping defined by the relation (4.1) can be represented by the hexagon in Fig. 4.3 (ii). Then we can show without loss of generality the actions of $A_{\rho}$ and $B_{\rho}$ are given as in Fig. 4.3 (ii) from the identification of the sides of $D_{o}\left(\Gamma_{\rho}\right)$. Moreover, the quadrilateral fundamental domain $D_{q}\left(\Gamma_{\rho}\right)$ is mapped to the period parallelogram in Fig. 4.3 (ii) which is determined by the lattice $\Omega=\Omega(\mu, \mu \rho)$ in the $u$ plane. These facts imply that there exist the correspondences $\mu \Leftrightarrow A_{\rho}$ and $\mu \rho \Leftrightarrow B_{\rho}$ which we used in order to show the correspondence $\rho \Leftrightarrow(3,3,3)$ (see §3).

REMARK. Theorem 4.1 comes from the work by $\mathrm{H}$. Cohn [C1]. The relation (4.1) is not unique, that is, we can have the same result by using the following relation:

$$
J(z)-1=\wp^{\prime}(u)^{2}=4 \wp(u)^{3}-1 .
$$

By using the same arguments as in the proof of Theorem 4.1 we obtain another example. In this case the images of the fundamental domains $D_{q}\left(\Gamma_{i}\right)$ and $D_{h}\left(\Gamma_{i}\right)$ are represented as in Fig. 4.4 (ii). 


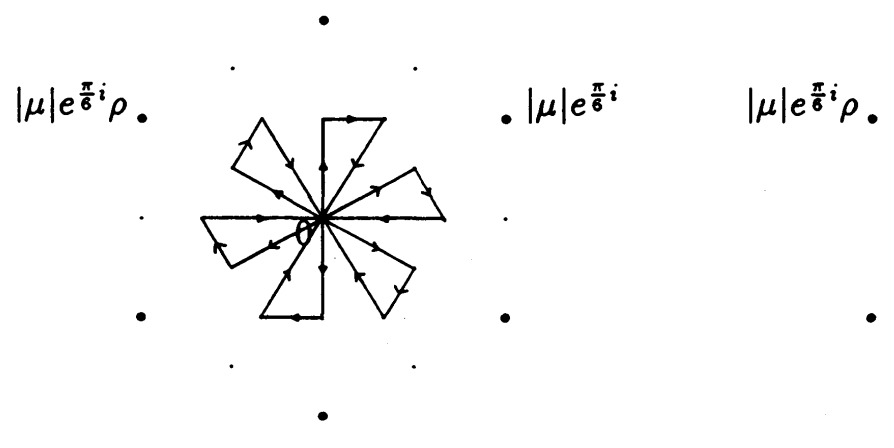

(i)

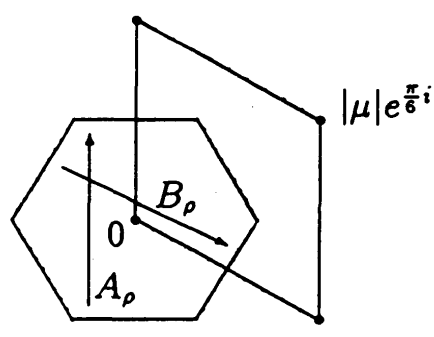

(ii)

(i) The segments $L$ ( $i \infty \rightarrow i \rightarrow \rho \rightarrow i \infty), L+j, j=-2,-1,1,2,3$ are mapped by the relation (4.1) to the segments $\left(0 \rightarrow\left((1 / 2)|\mu| e^{\pi i / 6}\right) e^{j \pi i / 3} \rightarrow((\sqrt{3} / 4)|\mu|) e^{j \pi i / 3} \rightarrow 0\right), j=0,1,2,3,4,5$.

(ii) The hexagon determined by $(\sqrt{3} / 4)|\mu| e^{j \pi i / 3}, j=0,1,2,3,4,5$ is the image of the octagonal fundamental domain by the relation (4.1).

FIGURE 4.3

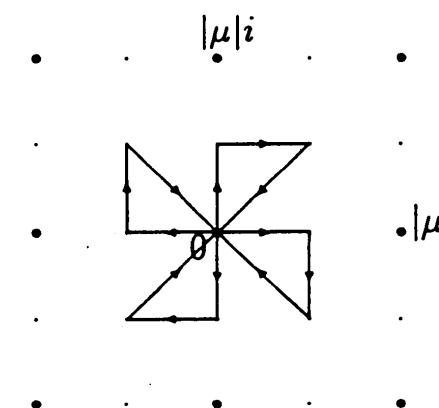

(i)

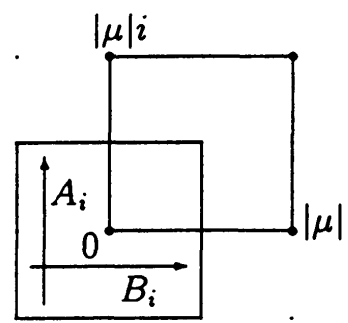

(ii)

(i) The segments $L_{i}\left(i \infty \rightarrow i \rightarrow \rho_{i} \rightarrow i \infty\right), L_{i}+\sqrt{2}, L_{i}-\sqrt{2}$ and $L_{i}-2 \sqrt{2}$ are mapped by the relations in (4.5) to the segments $(0 \rightarrow|\mu| / 2 \rightarrow|\mu|(1-i) / 2 \rightarrow 0),(0 \rightarrow-|\mu| i / 2 \rightarrow-|\mu|(1+i) / 2 \rightarrow 0)$, $(0 \rightarrow-|\mu| / 2 \rightarrow-|\mu|(1-i) / 2 \rightarrow 0)$ and $(0 \rightarrow|\mu| i / 2 \rightarrow|\mu|(1+i) / 2 \rightarrow 0)$.

(ii) The square determined by $\pm|\mu|(1+i) / 2, \pm|\mu|(1-i) / 2$ is the image of the hexagonal fundamental domain by the relations in (4.5).

FIGURE 4.4

THEOREM 4.2 (Cohn). The once punctured torus $(2 \sqrt{2}, 2 \sqrt{2}, 4) \in \mathcal{T}_{1,1}$ is mapped to the closed torus $i \in \mathcal{T}_{1,0}$ holomorphically by using the relations

$$
\wp^{\prime}(u)^{2}=4 \wp(u)^{3}-4 \wp(u) \text { and } J_{i}(z)=\wp(u)^{2} .
$$

The function $J_{i}$ in (4.5) is defined as follows:

PROPOSITION 4.1. We can construct a function $J_{i}$ which satisfies 
(i) $J_{i}$ maps $L_{i}$ onto $\mathbf{R}$ where $L_{i}=L_{i 1} \cup L_{i 2} \cup L_{i 3}$ and $L_{i 1}=L_{1}$,

$$
\begin{gathered}
L_{i 2}=\left\{\tau \in \mathbf{H}|| \tau \mid=1 \text { and }-\frac{\sqrt{2}}{2} \leq \operatorname{Re}(\tau) \leq 0\right\}, \\
L_{i 3}=\left\{\tau \in \mathbf{H}|| \tau \mid \geq 1 \text { and } \operatorname{Re}(\tau)=-\frac{\sqrt{2}}{2}\right\} .
\end{gathered}
$$

In particular, $J_{i}(i \infty)=\infty, J_{i}(i)=1$ and $J_{i}\left(\rho_{i}\right)=0$ where $\rho_{i}=e^{3 \pi i / 4}$.

(ii) $J_{i}$ maps $F_{i}$ onto $\mathbf{C}$ where $F_{i}=\{\tau \in \mathbf{H}|| \tau \mid \geq 1$ and $|\operatorname{Re}(\tau)| \leq \sqrt{2} / 2\}$.

(iii) The mapping $J_{i}: \mathbf{H} \rightarrow \mathbf{C}$ is holomorphic on $\mathbf{H}$.

For the proof of this proposition, we refer the reader to Chapter III in [H].

REMARK. Using the following relations instead of (4.5), we have the same result:

$$
\wp^{\prime}(u)^{2}=4 \wp(u)^{3}+4 \wp(u) \text { and } J_{i}(z)=-\wp(u)^{2} .
$$

\section{Construction of mappings.}

We proved in $\S 3$ that there exists the correspondence of the sets $L_{1} \Leftrightarrow M_{1}$. In this section we will show a construction of a holomorphic mapping between a once punctured torus in $M_{1}$ and a closed torus in $L_{1}$, which is obtained by modifying the construction in $\S 4$. In that construction fundamental domains for Fricke groups associated with $(3,3,3)$ and $(2 \sqrt{2}, 2 \sqrt{2}, 4)$ played very important roles, so we will display fundamental domains for Fricke groups in $M_{1}$.

We begin by recalling $M_{1}$ defined in $\S 3$ :

$$
\begin{aligned}
M_{1} & =\left\{(X, Y, Z) \in M \mid Z=\frac{1}{2} X Y\right\} \\
& =\left\{(X, Y, Z) \in M \mid(X, Y, Z)=\left(\frac{2 \sqrt{1+\alpha^{2}}}{\alpha}, 2 \sqrt{1+\alpha^{2}}, \frac{2\left(1+\alpha^{2}\right)}{\alpha}\right) \text { for } \alpha \geq 1\right\},
\end{aligned}
$$

where we introduce a parameter $\alpha \geq 1$ by setting $Y=\alpha X$ to simplify calculations.

The following arguments are due to [S].

DEFINITION 5.1. A Fricke group $\Gamma$ is called a special Fricke group if the associated coordinate $(X, Y, Z)$ of $\Gamma$ is in $M$.

Let $(X, Y, Z)$ be an associated coordinate of a special Fricke group $\Gamma=\langle A, B\rangle$. Then $A$ and $B$ are given by

$$
A=\left(\begin{array}{cc}
0 & -\frac{k}{X} \\
\frac{X}{k} & X
\end{array}\right) \quad \text { and } \quad B=\left(\begin{array}{cc}
Y-\frac{Z}{X} & -k \frac{Y}{X^{2}} \\
-\frac{Y}{k} & \frac{Z}{X}
\end{array}\right)
$$


for some $k>0$. In particular a fundamental domain for $\Gamma=\langle A, B\rangle$ is obtained by removing from the region

$$
\left\{z=x+i y \mid-k\left(\frac{3}{2}-\frac{X}{Y Z}\right) \leq x \leq k\left(\frac{1}{2}+\frac{X}{Y Z}\right) \text { and } y>0\right\}
$$

the isometric circles for the Möbius transformations $A, A^{-1}, B, B^{-1}, C=B^{-1} A^{-1}$ and $C^{-1}$.

From this, for $(X, Y, Z) \in M_{1}$ we obtain a special Fricke group $\Gamma_{\alpha}=\left\langle A_{\alpha}, B_{\alpha}\right\rangle$ with

$$
A_{\alpha}=\left(\begin{array}{cc}
0 & -1 \\
1 & \frac{2 \sqrt{1+\alpha^{2}}}{\alpha}
\end{array}\right) \quad \text { and } \quad B_{\alpha}=\left(\begin{array}{cc}
\sqrt{1+\alpha^{2}} & -\alpha \\
-\alpha & \sqrt{1+\alpha^{2}}
\end{array}\right) \quad \text { for } k=\frac{2 \sqrt{1+\alpha^{2}}}{\alpha}
$$

Then

$$
C_{\alpha}=B_{\alpha}^{-1} A_{\alpha}^{-1}=\left(\begin{array}{cc}
\frac{\alpha^{2}+2}{\alpha} & \sqrt{1+\alpha^{2}} \\
\sqrt{1+\alpha^{2}} & \alpha
\end{array}\right) \quad \text { and } \quad C_{\alpha} B_{\alpha} A_{\alpha}=\left(\begin{array}{cc}
-1 & -\frac{4 \sqrt{1+\alpha^{2}}}{\alpha} \\
0 & -1
\end{array}\right)
$$

By using the basic facts cited above, we represent a fundamental domain for $\Gamma_{\alpha}$ :

$$
\begin{aligned}
D_{h}\left(\Gamma_{\alpha}\right)= & \left\{z \in \mathbf{H}|| z+\frac{2 \sqrt{1+\alpha^{2}}}{\alpha}|\geq 1,| z+\frac{\sqrt{1+\alpha^{2}}}{\alpha} \mid \geq \frac{1}{\alpha},\right. \\
& \left.|z|>1,\left|z-\frac{\sqrt{1+\alpha^{2}}}{\alpha}\right|>\frac{1}{\alpha},-\frac{2+3 \alpha^{2}}{\alpha \sqrt{1+\alpha^{2}}} \leq \operatorname{Re}(z)<\frac{2+\alpha^{2}}{\alpha \sqrt{1+\alpha^{2}}}\right\},
\end{aligned}
$$

which is a hexagon whose sides are identified by actions of $A_{\alpha}, B_{\alpha}, C_{\alpha} B_{\alpha} A_{\alpha}$ and is called the hexagonal fundamental domain. We can introduce another fundamental domain which is a quadrilateral whose opposite sides are identified by actions of $A_{\alpha}, B_{\alpha}$ and is called the quadrilateral fundamental domain:

$$
\begin{aligned}
D_{q}\left(\Gamma_{\alpha}\right)= & \left\{z \in \mathbf{H}|| z+\frac{1+2 \alpha^{2}}{2 \alpha \sqrt{1+\alpha^{2}}} \mid>\frac{1}{2 \alpha \sqrt{1+\alpha^{2}}},\right. \\
& \left.\left|z+\frac{\alpha}{2 \sqrt{1+\alpha^{2}}}\right|>\frac{\alpha}{2 \sqrt{1+\alpha^{2}}},-\frac{\sqrt{1+\alpha^{2}}}{\alpha} \leq \operatorname{Re}(z) \leq 0\right\} .
\end{aligned}
$$

The quadrilateral fundamental domain (shown in normal outline in Fig. 5.1) can be identified with the once punctured torus $(X, Y, Z)$.

In order to construct the holomorphic mapping we use the following modified fundamental domain $D\left(\Gamma_{\alpha}\right)$ (the shaded part in Fig. 5.1): 


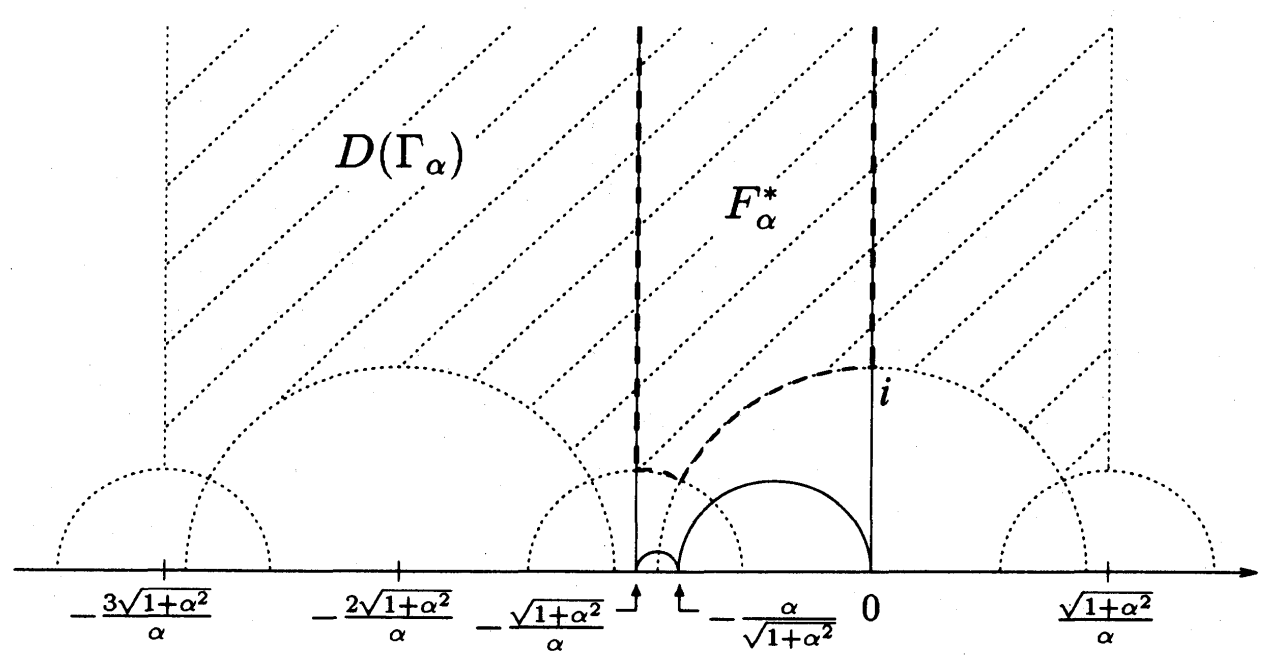

FIGURE 5.1

$$
\begin{gathered}
D\left(\Gamma_{\alpha}\right)=\left\{z \in \mathbf{H}|| z+\frac{3 \sqrt{1+\alpha^{2}}}{\alpha}\left|\geq \frac{1}{\alpha},\right| z+\frac{2 \sqrt{1+\alpha^{2}}}{\alpha}|\geq 1,| z+\frac{\sqrt{1+\alpha^{2}}}{\alpha} \mid \geq \frac{1}{\alpha},\right. \\
\left.|z| \geq 1,\left|z-\frac{\sqrt{1+\alpha^{2}}}{\alpha}\right| \geq \frac{1}{\alpha},-\frac{3 \sqrt{1+\alpha^{2}}}{\alpha} \leq \operatorname{Re}(z) \leq \frac{\sqrt{1+\alpha^{2}}}{\alpha}\right\} \ldots
\end{gathered}
$$

We introduce the following notations:

$$
\begin{gathered}
F_{\alpha}^{*}=\left\{z \in \mathbf{H}|| z+\frac{\sqrt{1+\alpha^{2}}}{\alpha}\left|\geq \frac{1}{\alpha},\right| z \mid \geq 1,-\frac{\sqrt{1+\alpha^{2}}}{\alpha} \leq \operatorname{Re}(z) \leq 0\right\}, \\
\eta=\left(-\frac{\sqrt{1+\alpha^{2}}}{\alpha}, \frac{1}{\alpha}\right) \quad \text { and } \zeta=\left(-\frac{\alpha}{\sqrt{1+\alpha^{2}}}, \frac{1}{\sqrt{1+\alpha^{2}}}\right) .
\end{gathered}
$$

The region $F_{\alpha}^{*}$ (shown in broken line in Fig. 5.1) is a quadrangle with angles $0, \pi / 2, \pi / 2$, $\pi / 2$.

PROPOSITION 5.1. We can construct a function $J_{\alpha}$ which satisfies the following:

(i) $J_{\alpha}$ maps $L_{\alpha}$ onto $\mathbf{R}$ where $L_{\alpha}=L_{\alpha 1} \cup L_{\alpha 2} \cup L_{\alpha 3} \cup L_{\alpha 4}$ and

$$
\begin{gathered}
L_{\alpha 1}=L_{1}, \quad L_{\alpha 2}=\left\{z \in \mathbf{H}|| z \mid=1 \text { and }-\frac{\alpha}{\sqrt{1+\alpha^{2}}} \leq \operatorname{Re}(z) \leq 0\right\}, \\
L_{\alpha 3}=\left\{z \in \mathbf{H}|| z+\frac{\sqrt{1+\alpha^{2}}}{\alpha} \mid=\frac{1}{\alpha} \text { and }-\frac{\sqrt{1+\alpha^{2}}}{\alpha} \leq \operatorname{Re}(z) \leq-\frac{\alpha}{\sqrt{1+\alpha^{2}}}\right\}, \\
L_{\alpha 4}=\left\{z \in \mathbf{H}|| z+\frac{\sqrt{1+\alpha^{2}}}{\alpha} \mid \geq \frac{1}{\alpha} \text { and } \operatorname{Re}(z)=-\frac{\sqrt{1+\alpha^{2}}}{\alpha}\right\} .
\end{gathered}
$$

In particular, $J_{\alpha}(i \infty)=\infty, J_{\alpha}(i)=P$ for some $P>0, J_{\alpha}(\zeta)=0$ and $J_{\alpha}(\eta)=-1$. 
(ii) $J_{\alpha}$ maps $F_{\alpha}$ onto $\mathbf{C}$ where

$$
\begin{gathered}
F_{\alpha}=\left\{z \in \mathbf{H}|| z+\frac{\sqrt{1+\alpha^{2}}}{\alpha}\left|\geq \frac{1}{\alpha},\right| z|\geq 1,| z-\frac{\sqrt{1+\alpha^{2}}}{\alpha} \mid \geq \frac{1}{\alpha},\right. \\
\left.-\frac{\sqrt{1+\alpha^{2}}}{\alpha} \leq \operatorname{Re}(z) \leq \frac{\sqrt{1+\alpha^{2}}}{\alpha}\right\} .
\end{gathered}
$$

(iii) The mapping $J_{\alpha}: \mathbf{H} \rightarrow \mathbf{C}$ is holomorphic on $\mathbf{H}$.

PROOF. We consider the transformations:

$$
\Sigma_{1}(z)=-\bar{z}, \quad \Sigma_{2}(z)=\frac{1}{\bar{z}}, \quad \Sigma_{3}(z)=-\frac{\sqrt{1+\alpha^{2}} \bar{z}+\alpha}{\alpha \bar{z}+\sqrt{1+\alpha^{2}}}, \quad \Sigma_{4}(z)=-\bar{z}-\frac{2 \sqrt{1+\alpha^{2}}}{\alpha} .
$$

These are reflections in the four circles, respectively:

$$
\Sigma_{1}: \operatorname{Re}(z)=0, \quad \Sigma_{2}:|z|=1, \quad \Sigma_{3}:\left|z+\frac{\sqrt{1+\alpha^{2}}}{\alpha}\right|=\frac{1}{\alpha}, \quad \Sigma_{4}: \operatorname{Re}(z)=-\frac{\sqrt{1+\alpha^{2}}}{\alpha} .
$$

They transform the upper half-plane into itself.

Let $\Gamma^{*}$ be a group generated by $\Sigma_{1}, \Sigma_{2}, \Sigma_{3}$ and $\Sigma_{4}$. The Fricke group $\Gamma_{\alpha}=\left\langle A_{\alpha}, B_{\alpha}\right\rangle$ is a subgroup of $\Gamma^{*}$ because $A_{\alpha}=\Sigma_{2} \Sigma_{4}$ and $B_{\alpha}=\Sigma_{3} \Sigma_{1}$. We obtain a complete and simple covering of the upper half-plane by the region $F_{\alpha}^{*}$ and its equivalents with respect to $\Gamma^{*}$. We note that neighborhoods of $i, \eta, \zeta$ and their equivalents are split into parts of four quadrilaterals equivalent to $F_{\alpha}^{*}$ under the reflections and that a neighborhood of $i \infty$ is split into parts of infinite number of quadrilaterals which are images of $F_{\alpha}^{*}$ under translations generated by $\Sigma_{1}$ and $\Sigma_{4}$.

By the Riemann mapping theorem there uniquely exists a mapping $J_{\alpha}: F_{\alpha}^{*} \rightarrow \mathbf{H}$ which is bijective, holomorphic and satisfies $J_{\alpha}(i)=P$ for some $P>0, J_{\alpha}(\zeta)=0, J_{\alpha}(\eta)=-1$ and $J_{\alpha}(i \infty)=\infty$. The function $J_{\alpha}$ can be analytically continued by the principle of reflection as follows:

$$
J_{\alpha}\left(\Sigma_{k} z\right)=\overline{J_{\alpha}}(z) \text { for } z \in F_{\alpha}^{*} \text { and } k=1,2,3,4,
$$

and we continue this process by iteration. Then continued $J_{\alpha}$ must be single-valued because the upper half-plane is simply connected and we can use the monodromy theorem.

LEMMA 5.1. We can assume that $P$ is larger than 1.

PROOF. We introduce the following notations:

$$
F_{\alpha}^{* \prime}=\Sigma_{4} F_{\alpha}^{*}, \quad \eta^{\prime}=\Sigma_{4} i, \quad \zeta^{\prime}=\Sigma_{4} \zeta, \quad L_{\alpha}^{\prime}=L_{\alpha 1}^{\prime} \cup L_{\alpha 2}^{\prime} \cup L_{\alpha 3}^{\prime} \cup L_{\alpha 4}^{\prime},
$$

where

$$
L_{\alpha 1}^{\prime}=L_{\alpha 4}, \quad L_{\alpha 2}^{\prime}=\Sigma_{4} L_{\alpha 3}, \quad L_{\alpha 3}^{\prime}=\Sigma_{4} L_{\alpha 2}, \quad L_{\alpha 4}^{\prime}=\Sigma_{4} L_{\alpha 1} .
$$

By using the same arguments as in the proof of Proposition 5.1, we can construct a function $J_{\alpha}^{\prime}: F_{\alpha}^{* \prime} \rightarrow \mathbf{H}$ which is bijective and holomorphic and maps $L_{\alpha}^{\prime}$ onto $\mathbf{R}$ with $J_{\alpha}^{\prime}(\eta)=$ $P^{\prime}$ for some $P^{\prime}>0, J_{\alpha}^{\prime}\left(\zeta^{\prime}\right)=0, J_{\alpha}^{\prime}\left(\eta^{\prime}\right)=-1$ and $J_{\alpha}^{\prime}(i \infty)=\infty$. The mapping $J_{\alpha}^{\prime}$ also can be analytically continued. Then $J_{\alpha}^{\prime}$ maps $F_{\alpha}^{*}$ onto the lower half-plane holomorphically and 
we have $J_{\alpha}^{\prime}(i)=-1, J_{\alpha}^{\prime}(\zeta)=0, J_{\alpha}^{\prime}(\eta)=P^{\prime}$. Therefore $J_{\alpha}^{\prime} \circ J_{\alpha}^{-1}$ is a holomorphic mapping between the extended planes which maps $\infty, P, 0,-1$ to $\infty,-1,0, P^{\prime}$, respectively. By a well-known theorem the cross-ratio of $(\infty, P, 0,-1)$ must be equal to the cross-ratio of $\left(\infty,-1,0, P^{\prime}\right)$, so we have $1 /(P+1)=P^{\prime} /\left(1+P^{\prime}\right)$ which means $P P^{\prime}=1$. Therefore we can assume that $P \geq 1$ without loss of generality.

Next, we consider an elliptic integral

$$
u(w)=\int_{0}^{w} \frac{1}{\sqrt{(w+1) w(w-P)}} d w
$$

If we set

$$
a(P)=\int_{0}^{P} \frac{1}{\sqrt{(w+1) w(P-w)}} d w \quad \text { and } \quad b(P)=\int_{P}^{\infty} \frac{1}{\sqrt{(w+1) w(w-P)}} d w,
$$

then we obtain $u(-1)=b(P), u(0)=0, u(P)=-a(P) i$ and that $u(w)$ is a holomorphic mapping from $\mathbf{H}$ to the interior of the rectangle determined by the four points $0, b(P),-a(P) i$ and $b(P)-a(P) i$. We recall that the two-sheeted covering of the extended complex plane with branch points $-1,0, P, \infty$ is a closed torus whose periods $\omega_{1}$ and $\omega_{2}$ are described by

$$
\omega_{1}=2 \int_{-1}^{0} \frac{1}{\sqrt{(w+1) w(w-P)}} d w \quad \text { and } \quad \omega_{2}=2 \int_{0}^{P} \frac{1}{\sqrt{(w+1) w(w-P)}} d w .
$$

Then we have $\omega_{1}=-2 b(P), \omega_{2}=2 a(P) / i$ and the ratio $\tau=\omega_{2} / \omega_{1}=a(P) i / b(P)$. Define $\tau(P)=a(P) i / b(P)$. We will show that $\tau(P)$ is uniquely determined by $P$.

LEMMA 5.2. $a(P)$ is monotone increasing with respect to $P$.

PROOF. The following equation is easily obtained by Cauchy's theorem

$$
\int_{-\infty}^{\infty} \frac{1}{\sqrt{(w+1) w(w-P)}} d w=0
$$

so we have

$$
\int_{-\infty}^{-1} \frac{1}{\sqrt{(w+1) w(w-P)}} d w=a(P) i
$$

Take two real points $P_{1}$ and $P_{2}$ with $0<P_{1}<P_{2}$. Since $0>(w+1) w\left(w-P_{1}\right)>$ $(w+1) w\left(w-P_{2}\right)$ for each $w$ with $-\infty<w<-1$, the following inequality holds

$$
\frac{i}{\sqrt{(w+1) w\left(w-P_{1}\right)}}>\frac{i}{\sqrt{(w+1) w\left(w-P_{2}\right)}} .
$$

Integrating both sides of the inequality with respect to $w$ from $-\infty$ to -1 , we have $i a\left(P_{1}\right) i>$ ia $\left(P_{2}\right) i$ which means $a\left(P_{1}\right)<a\left(P_{2}\right)$.

PROPOSITION 5.2. $\operatorname{Im}(\tau(P))$ increases monotonically with respect to $P$. 
Proof. In the definition of $b(P)$ we change the variable by $v=1 / w$, then

$$
b(P)=\int_{P}^{\infty} \frac{1}{\sqrt{(w+1) w(w-P)}} d w=\int_{0}^{\frac{1}{P}} \frac{1}{\sqrt{P}} \frac{1}{\sqrt{(v+1) v\left(\frac{1}{P}-v\right)}} d v=\frac{1}{\sqrt{P}} a\left(\frac{1}{P}\right) .
$$

Finally, we have

$$
\tau(P)=\sqrt{P} \frac{a(P)}{a(1 / P)} i .
$$

Since $a(P)$ is monotone increasing with respect to $P, \operatorname{Im}(\tau(P))$ is also monotone increasing with respect to $P$.

Now we have obtained that $\tau(P)$ is uniquely determined by $P$ and that if $P \geq 1$ then $\tau(P) \in L_{1}$ from $\tau(1)=i$. The values $g_{2}(\tau(P)), g_{3}(\tau(P))$ are real from Proposition 2.1 and the lattice $\Omega(1, \tau(P))$ is a real lattice from Proposition 2.2. By using the same arguments as in the proof of Proposition 2.8 we have the following facts with respect to the lattice $\Omega(1, \tau(P))$.

Proposition 5.3. For the lattice $\Omega=\Omega(1, \tau(P))$, we have

$$
\begin{array}{cc}
\wp(\mathbf{R}+\tau(P) / 2) \subset \mathbf{R} \cup\{\infty\}, & \wp(\mathbf{R}-\tau(P) / 2) \subset \mathbf{R} \cup\{\infty\}, \\
\wp(i \mathbf{R}+1 / 2) \subset \mathbf{R} \cup\{\infty\}, & \wp(i \mathbf{R}-1 / 2) \subset \mathbf{R} \cup\{\infty\} .
\end{array}
$$

As $J(\tau(P))>1$ the cubic polynomial $p(x)=4 x^{3}-g_{2}(\tau(P)) x-g_{3}(\tau(P))$ has three distinct real roots which will be denoted by $x_{1}, x_{2}, x_{3}$ with $x_{1}<x_{2}<x_{3}$.

LEMMA 5.3. Correspondences among points $-1,0, P$ and $x_{1}, x_{2}, x_{3}$ are described by using a linear transformation on $\mathbf{R}$, i.e., $x \mapsto\left(x_{2}-x_{1}\right) x+x_{2}$.

PROOF. We consider an elliptic integral

$$
v(w)=\int_{0}^{w} \frac{1}{\sqrt{4\left(w-x_{1}\right)\left(w-x_{2}\right)\left(w-x_{3}\right)}} d w .
$$

If we set

and

$$
c=\int_{x_{1}}^{x_{2}} \frac{1}{\sqrt{4\left(w-x_{1}\right)\left(x_{2}-w\right)\left(x_{3}-w\right)}} d w
$$

$$
d=\int_{x_{2}}^{x_{3}} \frac{1}{\sqrt{4\left(w-x_{1}\right)\left(w-x_{2}\right)\left(x_{3}-w\right)}} d w
$$

then $v(w)$ is a holomorphic mapping from $\mathbf{H}$ to the interior of the rectangle determined by $c, d$ and the two-sheeted covering of the extended complex plane with branch points $x_{1}, x_{2}, x_{3}, \infty$ is a closed torus whose ratio $\tau^{\prime}$ is equal to $d i / c$, which can be seen by the same arguments as the one we used in order to introduce $\tau(P)$. Then the ratio $\tau^{\prime}$ must be equal to the ratio $\tau(P)$ because the tori corresponding to $\tau^{\prime}$ and $\tau(P)$ are determined by the same real lattice $\Omega(1, \tau(P))$, which implies that we can take a holomorphic mapping between rectangles determined by $a(P), b(P)$ and $c, d$. By using Schwarz reflection principle we obtain the holomorphic mapping between the extended planes with $-1 \rightarrow x_{1}, 0 \rightarrow x_{2}, P \rightarrow x_{3}, \infty \rightarrow \infty$. 
Then the cross-ratio of $(-1,0, P, \infty)$ must be equal to the cross-ratio of $\left(x_{1}, x_{2}, x_{3}, \infty\right)$, so we have

$$
\frac{x_{2}-x_{1}}{x_{3}-x_{1}}=\frac{1}{P+1}
$$

which gives $x_{3}-x_{2}: x_{2}-x_{1}=P: 1$. Therefore we obtain the relation $x \mapsto\left(x_{2}-x_{1}\right) x+x_{2}$.

THEOREM 5.1. The once punctured torus determined by $\alpha$ is mapped to the closed torus determined by $\tau(P)$ holomorphically by using the relation

$$
\wp(u)=\left(x_{2}-x_{1}\right) J_{\alpha}(z)+x_{2} .
$$

PROOF. By the relation (5.1) the point $z=i \infty$ is mapped to a lattice point of the $u$ plane because of $J_{\alpha}(i \infty)=\infty$ and $\wp\left(u_{0}\right)=\infty$ for $u_{0} \in \Omega=\Omega(1, \tau(P))$. We can assume without loss of generality that the image of $z=i \infty$ by (5.1) is the point $u=0$. Suppose that $J_{\alpha}$ has a value $c_{0} \in \mathbf{C}$ with $c_{0} \neq-1,0, P$. Since for each $c \in \mathbf{C}, c \neq x_{1}, x_{2}, x_{3}, \infty$ the equation $\wp(z)=c$ has two simple solutions, for $J_{\alpha}=c_{0}$ we obtain two points of $u$ determined by the relation (5.1). The fundamental domain $D\left(\Gamma_{\alpha}\right)$ is a 2 -sheeted covering of the domain $F_{\alpha}$, so we can take two points which attain $J_{\alpha}=c_{0}$ in the fundamental domain $D\left(\Gamma_{\alpha}\right)$. Therefore the relation (5.1) gives correspondences between these two points in the $z$-plane and the two points in the $u$-plane. Now we consider the case where $J_{\alpha}(z)$ is real. We divide arguments into the following three cases.

(I) $J_{\alpha}(z) \geq P$, i.e., $z \in L_{\alpha 1}$.

Setting $J_{\alpha}(z)=c$, we have $\wp(u)=\left(x_{2}-x_{1}\right) c+x_{2}$. We can take a point $u$ with $\wp(u)=$ $\left(x_{2}-x_{1}\right) c+x_{2}$ on the line $\mathbf{R}$ because $\wp(\mathbf{R}) \subset \mathbf{R} \cup\{\infty\}$ and $\wp(u)$ is large positive if $u \in \mathbf{R}$ is

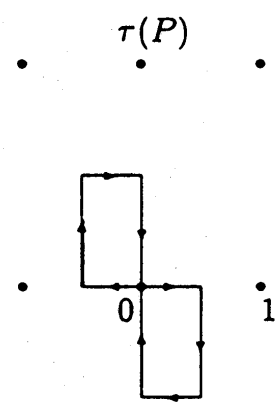

(i)

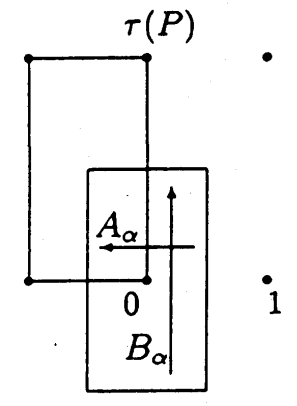

(ii)

(i) The segments $L_{\alpha}(i \infty \rightarrow i \rightarrow \zeta \rightarrow \eta \rightarrow i \infty)$ and $L_{\alpha}-\frac{2 \sqrt{1+\alpha^{2}}}{\alpha}$ are mapped by the relation (5.1) to the segments $\left(0 \rightarrow-\frac{1}{2} \rightarrow-\frac{1}{2}+\frac{1}{2} \tau(P) \rightarrow \frac{1}{2} \tau(P) \rightarrow 0\right)$ and $\left(0 \rightarrow \frac{1}{2} \rightarrow \frac{1}{2}-\frac{1}{2} \tau(P) \rightarrow-\frac{1}{2} \tau(P) \rightarrow 0\right)$. (ii) The rectangle determined by $\pm \frac{1}{2}(1+\tau(P)), \pm \frac{1}{2}(1-\tau(P))$ is the image of $D\left(\Gamma_{\alpha}\right)$ by the relation (5.1). 
small. If $J_{\alpha}(i)=P$ in (5.1) then $\wp(u)=\left(x_{2}-x_{1}\right) P+x_{2}=x_{3}$, which means that the point $u$ corresponding to $z=i$ must be ramification points $\pm 1 / 2$ in the $u$-plane. Therefore we can take segments $\left\{ \pm t \mid 0 \leq t \leq \frac{1}{2}\right\}$ as images of $L_{\alpha 1}$ by the mapping defined by the relation (5.1).

(II) $J_{\alpha}(z) \leq-1$, i.e., $z \in L_{\alpha 4}$.

Set $J_{\alpha}(z)=c$ then $\wp(u)=\left(x_{2}-x_{1}\right) c+x_{2}$. From Proposition 2.8 (i) we have $\wp(i \mathbf{R}) \subset$ $\mathbf{R} \cup\{\infty\}$ and if $u=i t, t \in \mathbf{R}$ is small then $\wp(u)$ is negative and $|\wp(u)|$ is large, so we can take a point $u$ with $\wp(u)=\left(x_{2}-x_{1}\right) c+x_{2}$ on the line $i \mathbf{R}$. Since $\wp(u)=x_{1}$ if $J_{\alpha}(\eta)=-1$ in (5.1), the point $u$ corresponding to $z=\eta$ must be ramification points $\pm \frac{1}{2} \tau(P)$ in the $u$-plane. Therefore we get segments $\left\{ \pm i t\left|0 \leq t \leq \frac{1}{2}\right| \tau(P) \mid\right\}$ as images of $L_{\alpha 4}$ by the mapping defined by the relation $(5.1)$.

(III) $-1 \leq J_{\alpha}(z) \leq P$, i.e., $z \in L_{\alpha 2} \cup L_{\alpha 3}$.

The point $u$ corresponding to $z=\zeta$ must be ramification points $\pm \frac{1}{2}(1-\tau(P))$ in the $u$-plane since $\wp(u)=x_{2}$ if $J_{\alpha}(\zeta)=0$ in (5.1). As the mapping defined by the relation (5.1) is conformal on $\mathrm{C} / \Omega$, we obtain Fig. 5.2 (i) by Proposition 5.3 and arguments in (I) and (II).

From Proposition 5.1 and the symmetry of the domain $F_{\alpha}$, the image of the fundamental domain $D\left(\Gamma_{\alpha}\right)$ by the mapping defined by the relation (5.1) can be represented by the rectangle including 0 in Fig. 5.2 (ii). Then we can take without loss of generality the actions of $A_{\alpha}$ and $B_{\alpha}$ as in Fig. 5.2 (ii) from the identification of the sides of $D\left(\Gamma_{\alpha}\right)$. Moreover, the quadrilateral fundamental domain $D_{q}\left(\Gamma_{\alpha}\right)$ is mapped to the rectangle in Fig. 5.2 (ii) which is determined by the lattice $\Omega=\Omega(1, \tau(P))$ in the $u$-plane. These facts imply that there exist the correspondences $\mu \Leftrightarrow A_{\alpha}$ and $\mu \tau(P) \Leftrightarrow B_{\alpha}$ for $\mu>0$ which we assumed in order to obtain the correspondence $L_{1} \Leftrightarrow M_{1}$ (see §3).

\section{References}

[Ab1] R. ABE, Abelianization of Fricke groups and rhombic lattices, Indag. Mathem., N.S. 11 (2000), 317-335.

[Ab2] R. ABE, Remarks on cusp forms for Fricke groups, to appear in Tokyo J. Math.

[Ah] L. V. AHLFORS, Lectures on Quasiconformal mappings, Van Nostrand (1966).

[C1] H. COHN, Approach to Markoff's minimal forms through modular functions, Ann. of Math. 61 (1955), 1-12.

[C2] H. COHN, Remarks on the cyclotomic Fricke groups, Lecture Notes in Math. 971 (1983), 15-23.

[F] R. FRICKE, Über die Theorie der automorphen Modulgruppen, Nachr. Akad. Wiss. Göttingen (1896), 91-101.

[H] E. HECKE, Lectures on Dirichlet Series, Modular Functions and Quadratic Forms, Vandenhoeck \& Ruprecht (1983).

[IT] Y. IMAYOSHI and M. TANIGUCHI, An introduction to Teichmüller spaces, Springer (1992).

[JS] G. A. Jones and D. Singerman, Complex Functions, Cambridge Univ. Press (1987).

[K] L. KEEN, On fundamental domains and the Teichmüller modular group, Contributions to Analysis, Academic Press (1974), 185-194.

[L] O. LEHTO, Univalent Functions and Teichmüller spaces, Springer (1987).

[MKS] W. MAgnUS, A. KARRASS and D. SolitAR, Combinatorial Group Theory, Interscience (1966).

[Mar] G. A. MARGULIS, Discrete Subgroups of Semisimple Lie Groups, Springer (1991).

[Mas1] B. MASKIT, Canonical domains on Riemann surfaces, Proc. Amer. Math. Soc. 106 (1989), 713-721. 
[Mas2] B. MASKIT, Parameters for Fuchsian groups II: topological type (1, 1), Ann. Acad. Sci. Fenn. 14 (1989), 265-275.

[R] M. RATNER, Rigidity of horocycle flows, Ann. of Math. 115 (1982), 597-614.

[S] A. L. SCHMIDT, Minimum of quadratic forms with respect to Fuchsian groups. I, J. Reine Angew. Math. 286/287 (1976), 341-368.

[W] S. WOLPERT, On the Kähler form of the moduli space of once punctured tori, Comment. Math. Helv. 58 (1983), 246-256.

[Z] R. J. ZIMMER, Ergodic Theory and Semisimple Groups, Birkhäuser (1984).

Present Address:

DEPARTMENT OF MATHEMATICS, KeIO UNIVERSITY,

YOKOHAMA, 223-8522 JAPAN.

e-mail: ryu2@math.keio.ac.jp 